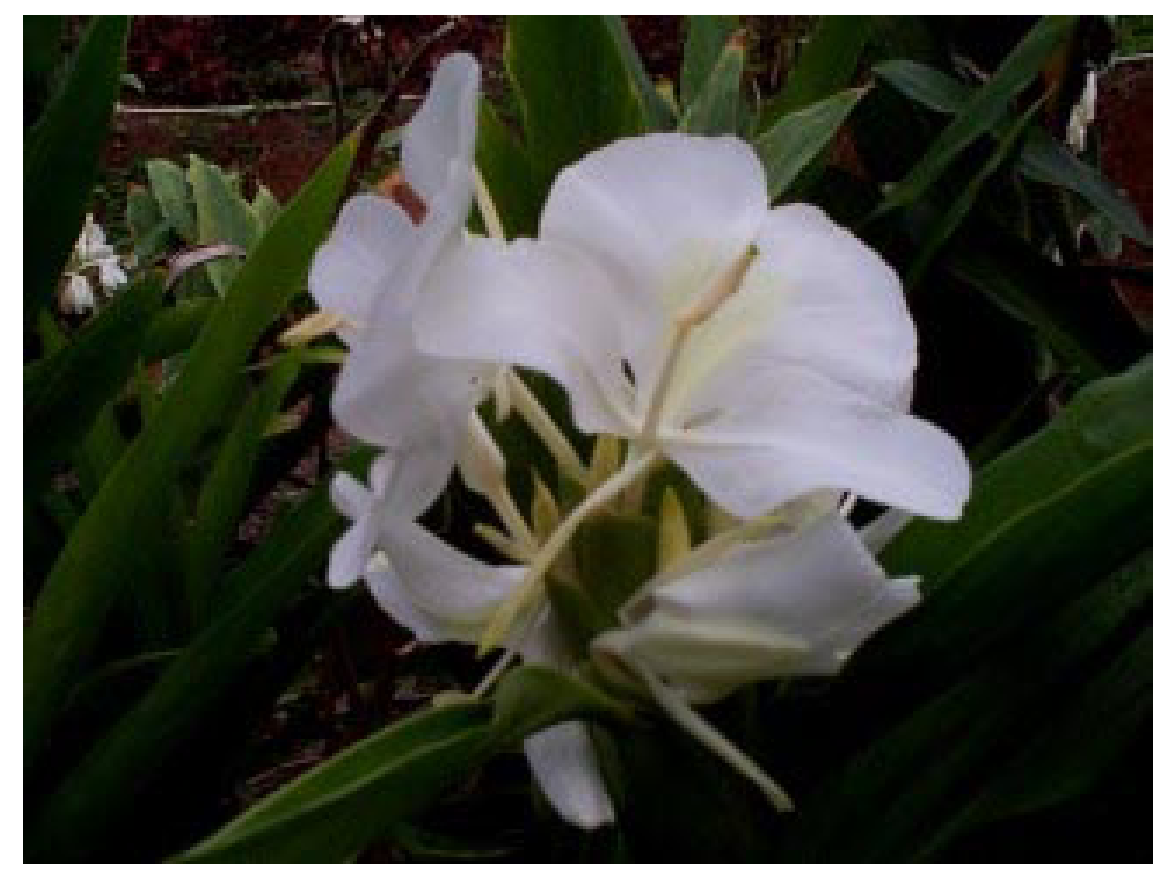

\title{
A User Manual for GINGER and its Post-Processor XPLOTGIN
}

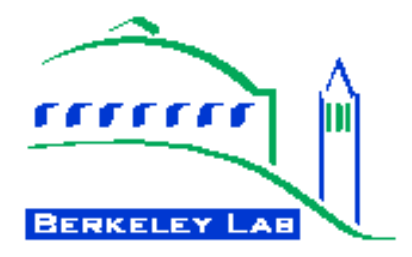

William M. Fawley

Lawrence Berkeley National Laboratory

Version 1.3f - December 2001

Report LBNL-49625 


\section{Acknowledgments}

The author has benefited from many useful discussions on simulation modeling from his colleagues in the general FEL community. In particular, he would like to acknowledge E.T. Scharlemann, J. Wurtele, P. Pierini, H.-D. Nuhn, K.-J. Kim, M. Xie, J. Eddighoffer, A. Zholents, Z. Huang, and S. Reiche as having contributed in one way or another to GINGER's development over the past 15(!) years.

Work on the GINGER simulation code has been supported by the Director, Office of Science, Offices of Basic Energy Sciences and High Energy and Nuclear Physics, of the U.S. Department of Energy under Contracts No. DE-AC03-76SF00098 to LBNL and DE-AC03-76SF0015 to SLAC. Computational resources have been provided in part by NERSC. The author also wants to thank the Accelerator and Fusion Research Division (AFRD) and the Center for Beam Physics (CBP) at LBNL and the Stanford Synchrotron Research Laboratory (SSRL) for support related to GINGER development.

\section{Copyright \& Distribution Restrictions}

(C) Regents of the University of California 2002. The Regents maintain legal copyright to the material contained in this document. However, this document may be freely copied and distributed for non-commercial uses and applications.

\section{Disclaimer}

NEITHER THE UNITED STATES DEPARTMENT OF ENERGY NOR THE LAWRENCE BERKELEY NATIONAL LABORATORY NOR THE REGENTS OF THE UNIVERSITY OF CALIFORNIA NOR ANY OF THEIR EMPLOYEES MAKE ANY WARRANTY, EXPRESS OR IMPLIED, OR ASSUME ANY LEGAL LIABILITY OR RESPONSIBILITY FOR THE ACCURACY, COMPLETENESS, OR USEFULNESS FOR THE SOFTWARE AND/OR DOCUMENTATION PROVIDED INCLUDING WITHOUT LIMITATION WARRANTY OF FITNESS OR CORRECTNESS FOR A PARTICULAR PURPOSE. 


\section{Contents}

Acknowledgments \& Legalese i

1 Introduction 1

2 General mechanics of obtaining and running the GINGER and XPLOTGIN codes 2

2.1 Hardware Portability . . . . . . . . . . . . . . . . . . . 2

2.2 Public Access to GINGER at NERSC . . . . . . . . . . . . . . . . . . . . . . . . 2

2.3 GINGER Execute Lines and Standard Options . . . . . . . . . . . . . . . . . . 3

2.4 Running GINGER on DOS/Windows Machines . . . . . . . . . . . . . . . . . . . . . 4

2.5 Running GINGER on Massively Parallel Processors at NERSC . . . . . . . . . . . 5

2.5.1 Cray-T3E Multiprocessor Execute Lines . . . . . . . . . . . . . . . . 5

2.5.2 IBM-SP Multiprocessor Execute Lines . . . . . . . . . . . . . . . . 6

2.6 Postprocessor XPLOTGIN Execute Line and Options . . . . . . . . . . . . . . . 6

3 GINGER "Modes" and Input File Details $\quad 8$

3.1 Overview of GINGER "Run Modes" . . . . . . . . . . . . . . . . . . . . 8

3.2 Namelist Input Information . . . . . . . . . . . . . . . . . . . . . . . . . 8

3.3 Electron Beam Input Variables . . . . . . . . . . . . . . . . . . . . . . 9

3.3.1 Macroparticle Number and Distribution . . . . . . . . . . . . . . . . . . 10

3.3.2 Electron Beam Emittance and Size . . . . . . . . . . . . . . . . . . . . . 10

3.3.3 Initial Tilts and/or Offsets in $x, x^{\prime}, y, y^{\prime} \ldots \ldots \ldots \ldots \ldots$

3.3.4 Instantaneous Energy Distributions and Chirping . . . . . . . . . . . 11

3.3.5 Pre-existing, Externally-produced Macroparticle Distributions . . . . . . . 12

3.3.6 "Quiet" Starts and Shot Noise . . . . . . . . . . . . . . . . . . . . . . . . . . . .

3.3.7 Random Number Seeds . . . . . . . . . . . . . . . . . . . . . . . 13

3.3.8 Longitudinal Current Profiles . . . . . . . . . . . . . . . . . . . . . . 14

3.4 Radiation Field Input Quantities . . . . . . . . . . . . . . . . . . . . . . . . . . . . 14

3.4.1 Optical Beam Size, Profile, and Waist Position . . . . . . . . . . . . . . . 14

3.4.2 Wavelength, Input Power, Slice \#, and Temporal Resolution . . . . . . . . 15

3.4.3 "Customizing" Time-dependent, Input Radiation Fields . . . . . . . . . . . 16

3.4.4 Saving to and Initializing from $z$-dependent Radiation Field Files . . . . . 17

3.5 Wiggler and Focusing Input Variables . . . . . . . . . . . . . . . . . . 18

3.5.1 Base Wiggler Input Parameters . . . . . . . . . . . . . . . . . . . . . 18

3.5.2 Constant $a_{w}(z)$ Wiggler Field $\ldots \ldots \ldots \ldots . \ldots \ldots$

3.5.3 Using a Predetermined Tapered Wiggler $a_{w}(z)$ Profile . . . . . . . . . . 18

3.5.4 Tapered Wiggler Self-Design in FRED-Mode . . . . . . . . . . . . . . . . 19 
3.5.5 Wiggler Focusing: Simple and Curved Poleface . . . . . . . . . . . . . . . 19

3.5.6 External Focusing: Continuous Quadrupoles and/or Ion Channels _. . . . 19

3.5.7 External Focusing: Discrete Quadrupole Magnet Lattices . . . . . . . . . . 20

3.5.8 Lattice Files, Wiggler and Quadrupole Errors, and Steering Corrections . . 21

3.5.9 Generation of Lattice File via the XWIGERR Program . . . . . . . . . . 21

3.5.10 Checking Beam Transport Properties through the Lattice . . . . . . . . . . 23

3.6 Waveguide, Space-charge, and External $E_{z}$ Input Parameters . . . . . . . . . . 23

3.6.1 Specification of Waveguide Properties . . . . . . . . . . . . . . . 23

3.6 .2 Longitudinal Space-charge . . . . . . . . . . . . . . . . . . . . . 24

3.6.3 External Accelerating/Decelerating Fields . . . . . . . . . . . . . . . 24

3.7 Drift Space and Optical Klystron Input Variables _ . . . . . . . . . . . . . . . 25

3.7 .1 Periodic Drift Spaces . . . . . . . . . . . . . . . . . . . . . . 25

3.7 .2 Optical Klystrons . . . . . . . . . . . . . . . . . . . . . . . . . . . 25

3.8 Oscillator Mode Input Variables ～. . . . . . . . . . . . . . . . . . . . 27

3.9 Grid and Numerical Integrator Input Parameters . . . . . . . . . . . . . . . . 27

3.9 .1 Simulation Grid . . . . . . . . . . . . . . . . . . . . . . . . . . 27

3.9 .2 Numerical Integrator Input Variables . . . . . . . . . . . . . . . . . . . 28

3.10 FRED-mode Parameter Scanning Capability . . . . . . . . . . . . . . . . . 28

3.10 .1 General Parameter Scanning Input Variables . . . . . . . . . . . . . 28

3.10.2 FRED-Mode Parameter Scanning with Multiple Processors at NERSC . . . 29

3.11 Output Diagnostics Control Variables . . . . . . . . . . . . . . . . . . . . . . . . 29

3.11 .1 Macroparticle Bunching Diagnostics . . . . . . . . . . . . . . . . . 30

3.11.2 Macroparticle Phase Space Snapshot Output . . . . . . . . . . . . . 30

3.11.3 Controlling the $z$-Frequency of Output to the Diagnostic Pltfile . . . . . . 31

3.12 Sample GINGER and XWIGERR Input Files . . . . . . . . . . . . . . . 31

3.12.1 Monochromatic, "Paladin" Tapered Wiggler Self-Design: inpalsD . . . 31

3.12.2 Paladin Sideband Growth in a Tapered Wiggler: inpalacSTD . . . . . . 32

3.12.3 Long pulse, LCLS 1.5A SASE x-ray FEL: inlcls_fodoSb . . . . . . . 34

3.12.4 Sample LCLS Wiggler Error Input File for XWIGERR Program . . . . . . 35

3.12.5 Example LCLS-case Template-File Use: inlcls-errA2 $\ldots . . . . \quad 36$

3.12.6 Short pulse, UCLA single-pass SASE expt.: inUCLAt2 . . . . . . . . 37

3.13 Names and Default Values for GINGER Input Namelist Parameters . . . . . . . . 38

4 "Preferences" File for the XPLOTGIN Post-Processor 46

4.1 General Information . . . . . . . . . . . . . . . . . . . . . 46

4.2 Graphics Output Device, Color, "Logo" Control Variables . . . . . . . . . . . . 46

4.3 Pltfile Input Read Control Variables . . . . . . . . . . . . . . . . . . . . . . . 47

4.4 Radiation Power and Bunching Plot Control Variables . . . . . . . . . . . . . . . 47 
4.5 Spectrum Plot Control Variables . . . . . . . . . . . . . . . . . . . . 48

4.6 Generating ASCII Output Tabular Data Files . . . . . . . . . . . . . . . . . . . . . 49

4.7 Generating SDDS Format Output Files . . . . . . . . . . . . . . . . . . . . . . . . 49

4.8 Generating "Special Purpose" SDDS Output Files . . . . . . . . . . . . . . . . 50

4.9 Generating HDF Output Data Files . . . . . . . . . . . . . . . . . 50

4.10 Generating Wiggler Exit, Radiation Field Dump Files . . . . . . . . . . . . 50

4.11 Generating $z$-dependent, Single-Slice, Radiation Field Dump Files . . . . . . . . 51

4.12 Macroparticle Phase Space Plot Control Variables . . . . . . . . . . . . . . . . . 51

4.13 Default Values for Preference File Namelist Variables . . . . . . . . . . . . . . . 52

5 The Physics Model of GINGER

5.1 Application of the Paraxial Wave Equation . . . . . . . . . . . . . . . 55

5.2 Application of the KMR Equations . . . . . . . . . . . . . . . . 56

5.3 GINGER's Transverse Macroparticle Mover . . . . . . . . . . . . . . . . . . 56

5.4 Temporal Structure of GINGER . . . . . . . . . . . . . . . . . . . 56

5.5 Discrete Slippage Model . . . . . . . . . . . . . . . . . . . . . . . . . 57

5.6 Temporal/Frequency Window Duration and Resolution Considerations . . . . . 58 


\section{Introduction}

This manual is intended to give a brief introduction to the physics and necessary input parameters relevant to the two-dimensional, polychromatic FEL simulation code GINGER and its graphical postprocessor XPLOTGIN. The manual presumes that the interested reader has a reasonably thorough knowledge of FEL physics, including those of time-dependent (i.e. polychromatic) effects such as sideband generation and self-amplified spontaneous emission emission (SASE). Throughout the following text, input parameter names are typeset in red bold Courier font, input parameter values in blue, and user input to the console in green. Since the first version of this manual was written in 1996, GINGER and its postprocessor have been extensively rewritten in Fortran90 and have become more modularized to aid in porting to different hardware platforms. Additional features, such as the ability to exploit (in certain situations) the multiple processor capabilities of the Cray J90's and the massively parallel Cray T3E and IBM SP machines at NERSC, have been added. This version of the user manual also documents new physics features (e.g. FODO focusing, wiggler errors), input variables, and postprocessor capabilities which have been added in the last couple years, including changes up to the 1 December 2001 code versions.

GINGER is a direct descendent of FRED, the original LLNL 2-D FEL simulation code. FRED was a single-pass amplifier particle-in-cell (PIC) code which modeled the interaction between electrons in one ponderomotive well and a monochromatic, $r$ - and $z$-dependent electromagnetic wave. By monochromatic, we mean that all field quantities (and many particle quantities such as the par-

ticle bunching) vary exactly as $\exp \left(-i \omega_{o} t\right)$. Other quantities such as beam current and energy are presumed to be time-invariant over "slow" time scales (i.e. when averaged over $\sim$ dozens of wave periods). Hence, FRED and its monochromatic descendents (e.g. FRED3D and the harmonic code NUTMEG) are useful in modeling FEL's where shot noise, slippage, current and energy variations, and sideband growth may be neglected.

Originally, GINGER was developed in the mid-1980's to examine the consequences of sideband growth in single-pass amplifiers. Soon after, a shot noise package was added to examine the minimum excitation level of sidebands and to model SASE growth in the LLNL microwave FEL experiments ELF (35-GHz) and IMP (140- and 250-GHz). In the early 1990's, GINGER began to be used for both x-ray SASE FEL studies (LCLS at SLAC and TESLA-FEL at DESY) and for modeling some of the longer wavelength proof-of-principle SASE experiments which have been done at UCLA/LANL, Brookhaven, and Argonne. In the last couple years modifications to GINGER have been primarily directed toward increasing platform independence and giving it greater ability to model more exactly actual experimental conditions. GINGER remains a work in progress and the user should check for recent additions/changes via the README and CHANGES files in the NERSC public archive space (see $\S 2.2$ ).

The remainder of this manual is organized as follows. Section 2 gives instructions on both how to obtain and how to run GINGER and its post-processor XPLOTGIN while Section 3 gives details 
concerning the types of runs possible and the input file variables which define and control a specific GINGER run. A number of sample input files are shown in $\S 3.12$ which will help a beginning GINGER user get up and running. Section 4 describes the post-processor and a user-modifiable "preferences" file which control the types and details of graphical and text output. Section 5 describes GINGER's physics, structure, underlying assumptions, and thus limitations.

\section{General mechanics of obtaining and running the GINGER and XPLOTGIN codes}

\subsection{Hardware Portability}

GINGER and its graphics postprocessor XPLOTGIN are written in Fortran90 and are targeted (preferentially) toward UNIX platforms. Access to and use of GINGER are most easy if the user has an account at NERSC (National Energy Research Supercomputer Center), which is funded by the Office of Science in the U.S. Department of Energy.

Alternatively, since both codes compile, link, and run on most UNIX workstations with standard F90 compilers, arrangements can be made with serious users for executables to be supplied for running on their own desktop computers. Successful ports of GINGER have also been made to Windows NT (Alpha/DEC F90 compiler), DOS-Windows95/98/ME (Lahey F95 compiler), and Linux (X86/Redhat/Portland Group F90 compiler and Alpha/DEC F90 Compiler). However, the multipass oscillator capability in GINGER currently relies on a few matrix subroutines from the commercial IMSL libraries, which may pose access problems for some users. For this and other reasons, it is suggested that one run at NERSC.

The postprocessor relies upon a set of graphics subroutines which are built on top of the NCAR graphics libraries. Nearly all these routines have equivalents in other graphics libraries (e.g. CERNLIB) and could be ported by a "hungry-"enough user. Some output routines in XPLOTGIN also rely upon the publicly available HDF library from NCSA. An experimental version of the post-processor which substitutes tabular output (e.g. SDDS files; see $\S 4.6$ and $\S 4.7$ ) for graphics is available for those platforms (e.g. Windows/DOS, LinuxX86) without NCAR graphics capability. At present, source code for XPLOTGIN is available from the author. However, source code is normally not available for GINGER due both to U.S. Government export control considerations, and the author's desire to avoid the generation/proliferation of incompatible and possibly buggy versions.

\subsection{Public Access to GINGER at NERSC}

At NERSC, executables for both GINGER and XPLOTGIN, together with some sample input files may be copied (using the system hsi program) from the publicly-readable HPSS directory named 
/nersc/mp40/fawley/pub. Currently, executables for the IBM-SP, Cray-J90 and T3E, DOS/Windows(X86) and Linux(X86) should be available from this directory. Since NERSC seems to change its HPSS directory structure surprisingly often, you might need to contact me (e-mail: fawley@lbl.gov) if you have problems locating the current equivalent of this directory. The pub directory contains both a README file with information concerning other files in the directory and also a CHANGES file which attempts to list the more important modifications made to GINGER and XPLOTGIN. I will attempt to keep both older versions of these codes (in the /OLD subdirectory) and reasonably "fresh" ones with more current modifications. Please e-mail me if you have problems with either access or version compatibility.

Given the retirement of the Cray C90, one should use either the Cray J90, IBM-SP (both serial and MPP versions), or Cray T3E executables. From a speed point of view, the IBM-SP is so much faster than any of the other NERSC machines that it is by far my preferred platform (moreover, the postprocessor runs on it too). When running the postprocessor at NERSC (especially under "batch" mode), be sure that the list of your loaded "modules" includes the NCAR package. Use the module list command to check which modules are actually loaded; use module load ncar if "NCAR" is not listed.

\subsection{GINGER Execute Lines and Standard Options}

Ignoring temporarily multiprocessor runs on MPP platforms, the execute command line to begin a GINGER run is: $\quad x g i n g e r$ run_name [Options]

Typing $\mathrm{xg}$ inger alone or $\mathrm{xg}$ inger $-\mathrm{h}$ will echo to the user console some simple help instructions and the source version. Beginning in October 2001, the $-r$ run name string must be given on the execute line. Here run name is a 1-24 character alphanumeric string (e.g. "elfaa"); it should not start with a dash("-") nor contain characters such as "*”" which could play havoc with the system shell. The run_name both identifies the run and acts as a substring contained within the name of various disk files GINGER creates (e.g. "pltelf3a") or reads. If the input file is not specified by the $-i$ option, GINGER presumes that a proper input file named inrun_name (e.g. inelf3a) exists within the directory from which GINGER is being run. Likewise, if the run employs a tapered wiggler (i.e. the input deck contains idesign $=0$ and $\mathbf{l c n s t w g l = . f}$ ) and the -b option is not used, GINGER presumes that a tapered wiggler file (generally referred to as a bwfile) exists with the name bwrun_name (e.g. bwelf3a).

The various execute line options include:

-i infile Here infile is the name of the input file which will be read by xginger and will override the name corresponding to the run_name.

$-\mathrm{b}$ bwfile Here bwfile is the name of the tapered wiggler field file containing $a_{w}(z)$ to be either read (idesign=0) or to be created (idesign=2) (only possible when running in 
monochromatic FRED-mode).

-t template_file The -t option is newly available in October 2001. A template_file is a "normal" GINGER input file which contains the usual header lines and namelists. It is read before the usual input file (whose name is set by the run_name or $-i$ option). The template_file should be used to set various common input parameters for a general class of runs (e.g. standard LCLS parameters). The normal input file is subsequently read and can be used to override some of the specific namelist variable values set by the template file and/or set additional values. For those users of the object-oriented mindset, the template file can be thought of as a "base class" whose namelist variable values the normal input file "inherits" and then can optionally extend.

For example, the template file might have ntest $\mathrm{p}=2048$ but the input file might set ntest $p=8192$, nfold_sym=12, and nhar_io $=135$ in order to examine fifth harmonic bunching properties. The "final" values actually used in the GINGER run will be properly echoed to the pltfile output data file. An example using templates is given in $§ 3.12 .5$.

-f field_file A field_filecontains radiation field information from a previous run and can be used to initialize such in special types of GINGER runs (see $\S 3.4 .4$ for details).

$-\mathrm{h}$ As mentioned above, this option leads to GINGER printing out some simple "help" instructions to the user console and then exiting.

As GINGER initializes, it first echoes to the user console the underlying source version

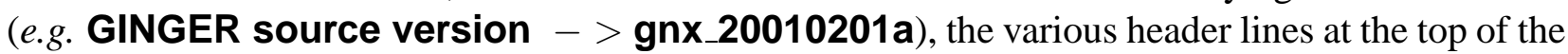
input file, and then some general characteristics of the electron beam, radiation field, and magnetic wiggler. While running, GINGER creates a so-called pltfile (e.g. pltelfa3a) containing the output field and particle diagnostics which will be subsequently analyzed by the postprocessor. This file is currently in ASCII format and can exceed 10 megabytes if nside, the number of electron beam slices, is large (e.g. $\geq 192)$. When running with periodic boundary conditions, a binary format parfile may be created for temporary storage of particle information. When GINGER is run in monochromatic "FRED" mode (lfred=.t) and is instructed to dump out macroparticle phase space information at various $z$-locations via the input variable nspec $>0$, a binary format spcfile is created (e.g. speclfa3a) which can be read by the post-processor to create macroparticle scatter plots (see $\S 4.12$ ). When requested (via the 1 debug=. $t$ input switch), GINGER can also create an ASCII debugfile which is a catch-bag of normally obscure numerical integrator diagnostics.

\subsection{Running GINGER on DOS/Windows Machines}

Although the author frowns upon usage of operating systems from the Evil Empire of Redmond, such occurs unfortunately (even by supposedly intelligent FEL scientists who should know better!). GINGER will now run under Windows via a DOS command window/terminal. I strongly suggest 
getting the free Cygwin package which provides a reasonably robust UNIX-like environment. Presuming you have obtained an executable named xginger.exe, you would run it with the exact same command line as on a UNIX box except obviously replace the name "xginger" with "xginger.exe" (of course, you could always make a soft link under Cygwin to allow you to use the name "xginger"). Presently (late 2001), only tabular ASCII output (i.e. no graphics) is supported under the DOS/Windows version of the post-processor. However, in single slice FED-mode, GINGER itself can create a simple datfile containing simple ASCII tables of items such as radiation power, rms delta gamma, microbunching fraction $b$ versus $z$ via the input switch 1_datfile. This switch is defaulted to .true. for the DOS and Linux executable versions. One can then use gnuplot or Excel to plot this file directly without having to use the post-processor at all.

\subsection{Running GINGER on Massively Parallel Processors at NERSC}

GINGER was ported to the NERSC Cray-T3E (mcurie.nersc.gov) in late 1999 and IBM-SP (seaborg.nersc.gov) in summer 2000. As of fall 2001, the author believes the MPP versions are quite robust. Executables are available for both platforms in the the author's public HPSS file space (see §2.2). GINGER can effectively use multiple processors for the following types of runs: (1) In polychromatic mode, both "long-pulse" runs (i.e. periodic boundary conditions in time) and (as of spring 2001) short pulse "transit-time" (non-periodic BC) runs. (2) In monochromatic FRED-mode, multiple slice, "parameter-scanning" runs (see §3.10). In serial (i.e. single-processor) mode, all run modes of GINGER should work properly: monochromatic single slice and multi-slice parameterscanning FRED-mode; single- and multipass, monochromatic and polychromatic oscillator mode; polychromatic short pulse "transit-time" and periodic BC "long-pulse" modes. Tests by H.-D. Nuhn of SLAC on the Cray-T3E for LCLS-type runs have shown nearly exact linear speed-up as the number of processors used increased from 2 to 64 .

\subsubsection{Cray-T3E Multiprocessor Execute Lines}

To run in multiprocessor mode on the Cray-T3E, the user should precede the normal execute line (i.e. $x g$ inger $r=\ldots$ ) with the phrase mpprun $-\mathrm{n}$ NPROC where $N P R O C \geq 1$ is the number of T3E processors requested. NPROC should be an integer factor of nside, the number of electron beam slices in the run. For example, when nside $=64$, permitted choices for $\mathbf{N P R O C}$ are 2, 4, 8, 16, 32, 64 but not 3, 17, 24, 36, etc. . At present, GINGER creates NPROC separate output pltfiles, numbered 000 and up. For example, mpprun $-\mathrm{n} 8$ xginger $r=$ palac will create 8 separate pltfiles, whose names begin with plt000palac and end with plt007palac. For later analysis by the postprocessor, these pltfiles must be concatenated together into one big, single file. The plt000... file also must be at the head of the resultant concatenated file. The UNIX cat command is probably the simplest way to do this: e.g. cat plto*palac > pltpalac will put all the 
subfiles together in the correct order. As of early 2002, the NCAR libraries DO exist on the T3E and the postprocessor has been successfully compiled and linked. Consequently, it is no longer necessary for the user to upload the GINGER-made pltfiles to other platforms for post-processing.

\subsubsection{IBM-SP Multiprocessor Execute Lines}

Multiple processors can also be used effectively to run GINGER on the IBM-SP. Since the IBM-SP version of GINGER (which contains "mpp" in its name) in the public HPSS directory was compiled to be "MPP-ready" (i.e. one does not need to use "POE"), one uses a normal (i.e. single processor) execute line for GINGER together with the additional phrase -procs NPROC where, as with the T3E, NPROC is the number of processors requested. With the arrival of the newest version of the IBM-SP (seaborg.nersc.gov) which is composed of 16-processor SMP nodes, it is most sensible to run in MPP mode with multiples of 16 processors (in any case, you will be charged for the full 16 processors of each node). As with the Cray-T3E, after a GINGER MPP run completes, one must concatenate the various pltfiles into one large one with the cat command to be readable by the postprocessor. The IBM-SP supports NCAR graphics and a version of the post-processor XPLOTGIN is available via hsi access of HPSS. Consequently, it is not necessary to export output pltfiles to another machine.

\subsection{Postprocessor XPLOTGIN Execute Line and Options}

The execute line for the postprocessor XPLOTGIN is:

xplotgin -r run_name [OPTIONS]

The postprocessor presumes that a file named pltrun_name (e.g. pltelf3a) exists in the working directory. Presuming the processor can generate graphics (which presently is not true for the Windows/DOS and Linux versions), the user may choose a particular output device/file by optionally adding to the XPLOTGIN execute line an uppercase mnemonic which may take one of the following values: $\mathrm{X} 11$ for direct output to an X-windows screen, CGM to generate a CGM graphics file, or POST to generate a Postscript file. When running at NERSC, the default graphical output goes to a CGM file named run_name.cgm (e.g. elf3a.cgm). On UNIX workstations, the default is X11 output which leaves no residual file when XPLOTGIN finishes. Consequently, the user should use CGM or POST instead if one expects to look at or use the output later. A "preferences" file, if present in the local working directory, will be read and can be used to control various plotting options and the generation of additional output files (e.g. SDDS format files) for further analysis. The default name for the preferences file is xplotgin.pref but this name can be optionally overridden by typing on the XPLOTGIN execute line -pref pref_file. Since since many specialized features of the post-processor are now controlled by the preferences file, the user should make the effort to master its usage (see $\S 4.1$ for more details). 
Significant effort has been spent to ensure upward compatibility of pltfiles from "old" GINGER runs with new versions of the post-processor. Please communicate any compatibility problems encountered in this direction (new postprocessor executable aborting when analyzing an old pltfile). On the other hand, old versions of the post-processor can be seriously incompatible with pltfiles created by more recent versions of GINGER both because the data format in the pltfiles occasionally changes (e.g. new variables are written out) and because occasionally new variables are added to the \&POSTPROC namelist located near the begining of the pltfile.

The user can use one or more of the NCAR family of ctrans applications to view output CGM files and to plot individual frames to various device drivers such as X11, Postscript, etc.. The idt program is particularly useful on an X11 display-server as one can scroll through the frames in a given CGM file, examine multiple frames from one or more CGM files simultaneously, and do some rudimentary animation on screen. The ghostview program is useful for examining Postscript output on an X11 screen.

If, due to a user mistake or whatever, either GINGER or the XPLOTGIN tries to open an input or other such file which is not present on disk, an error message will be sent to the terminal and the user can type in a new name. Alternatively, the user can type end and the code will exit. Obviously, if one runs the code in "background" or in batch mode, recovery from such an such an error is difficult, if not impossible. Under UNICOS, one should kill the process (i.e. kill -9 pid where pid is the process number). Typical run times for the post-processor are of order one minute or less but can become larger if the pltfile is huge (i.e. $\geq 20 \mathrm{MB}$ ) or significant post-processing is needed (e.g. far-field mode calculations). 


\section{GINGER "Modes" and Input File Details}

\subsection{Overview of GINGER "Run Modes"}

Over the past decade, GINGER has evolved from a code targeted toward modeling sideband growth in single pass amplifiers to a beast with far greater pretensions. The author considers these as "run modes" and they may be broken down into various classes via different criteria. The first criteria is monochromatic simulation (known as "FRED mode" which is set by the input variable lfred=.t) versus polychromatic simulation ("time-dependent mode" with lfred $=. f$ ) involving a discrete band of wavelengths centered upon a central, usually resonant wavelength.

A second criteria is the type of FEL configuration. Normally, GINGER models single-pass devices but is also capable of modeling (in both FRED- and time-dependent modes) multi-pass oscillators (§3.8) and (with a fair amount of effort) more complicated configurations (e.g. oscillatorradiator combinations [\$3.7.2], harmonic "cascade" multiple undulators). Both drift space and optical klystron sections may comprise part of the undulator.

Within time-dependent mode, the user chooses either the default "long-pulse" mode, in which case periodic boundary conditions are applied in time, or "short-pulse" mode (via the input variable ltransit $=. t)$, in which case the temporal window normally includes the entire electron beam pulse (+ slippage) and the user must specify a longitudinal current profile. There are also options to create and use various types of "restart" files; however, beginning GINGER users are advised to postpone their use.

Within FRED-mode, the user normally models single pass amplifier configurations. However, one can also model multi-pass oscillators and also use special restart radiation field files created by time-dependent runs ( $\$ 3.4 .4)$. One can vary a "scanning" parameter ( 33.10$)$ to study output power sensitivity to parameters such as electron beam current or undulator pole strength.

\subsection{Namelist Input Information}

GINGER uses the "namelist" capability of Fortran which (hopefully) minimizes the work required to keep a given input file "runnable". The Fortran90 language has many, many positive features relative to Fortran77 but its present namelist capability seems "buggy" on many compilers (in particular the Sun) and is not as robust as one would prefer. In particular, it is no longer possible to put comments within the NAMELIST body as was possible for GINGER under the CFT77 compiler at NERSC. Fortran90 also prefers that the namelist identifier be preceded by an ampersand (e.g. \&in) rather than a dollar sign, and that the end of the namelist be specified by a slash (e.g. "/END") (although, depending upon the F90 compiler, dollar signs may work in both cases). On some platforms, depending upon which version of the F90 compiler is used, when giving input to set a one-dimensional multi-element array (i.e. vector) it may be necessary to specify indices. For 
example, iseeds $(1: 2)=342845$ 663857. Many F90 compilers (e.g. NERSC Crays) have quite limited ability to handle arrays of "TYPE" structures in F90 namelists. This limitation has forced some rather inelegant (i.e. ugly) coding for inputting items such as periodic drift spaces.

The beginning of each GINGER input file (various examples are shown in §3.12) must contain a group of informational header lines whose essential purpose is to be repeated verbatim in the first frame of the postprocessor output. These header lines can help remind the user as to what was so incredibly special or important about this particular set of input parameters. The header must be at least one and no more than 10 lines long; its end is indicated by the presence of a "\$" symbol. Following the header lines is the first (and, for most runs, only) namelist identifier "\& in". Note that this identifier must begin in the second column (the Fortran namelist structure prohibits any symbol from appearing within the first column). The namelist should terminated by "/END", also beginning in the second column. Most compilers permit a simple "/" but for readability "/END" is much safer. Likewise, each namelist line may contain multiple input variables, but for readability one should use this capability sparingly. As explained later, when simulating optical klystron configurations (§3.7.2), multipass oscillators ( 33.8$)$ and/or certain other situations, the input file will need a second namelist named in_extra.

If an error is encountered in the namelist, further reading ceases, a full dump of namelist variables and their current values (i.e. when the input error was first encountered) are written out to the user's terminal window, and the program aborts. Careful inspection of the dumped parameter values versus what was attempted to be input might help illuminate the namelist error (once again, the namelist implementation in Fortran90 is surprisingly brain dead - one hopes that future versions will directly indicate the source of the error in the namelist read). Common errors in namelist input include specifying floating point values for integer variables (e.g. nside $=32.0$ rather than nside=32), ASCII variables (i.e. strings) for reals or integers (e.g. nside=' $32.0^{\prime}$ ), arrays for scalars (e.g. omgj $=0.330 .45)$, and plain vanilla typos for variable names (e.g. nsise=32).

Please note that for "historical" reasons, nearly all variables involving transverse dimensions (e.g. e-beam size) should be given in units of centimeters while those associated with longitudinal dimensions (i.e. wiggler wavelength) require units of meters. In a few cases, certain longitudinal variables require units of Rayleigh ranges but for nearly all of these, there are corresponding alternative input variables for which meters are used (e.g. zmaxsim is in Rayleigh ranges while zmxmeter is in meters). Also, in cases such as transverse emittance and Twiss parameters where the general accelerator community normally uses MKS units, GINGER attempts to follow suit.

\subsection{Electron Beam Input Variables}

Setting up the electron beam for a very simple, standard GINGER run requires that the user need only specify the beam current in Amperes (current), the MKS normalized emittance in rad-m (emit mks), the beam energy in $\mathrm{MeV}$ (energy). Normally, one also gives the number of beam 
slices (nside) to be simulated in either polychromatic mode (default value $\mathbf{n s i d e = 4}$; see $\S 3.4 .2$ ) or monochromatic "FRED" mode (lfred=.t has a default of nside $=1$ ). The other variables will be set to default values which will result in a beam loaded in equilibrium with the wiggler focusing, a uniform ellipsoid distribution in 4-D transverse phase space, and representation by 1024 macroparticles per slice. Usually, the user will want to set many other variables and we discuss the most important ones in the following paragraphs.

\subsubsection{Macroparticle Number and Distribution}

GINGER uses a moderate number (ntestp) of macroparticles (usually 512-8192 is adequate) per slice to represent the actual electrons in each beam slice. The default macroparticle load has now been changed (November 2001) to a Gaussian (jmg=+2). Alternative macroparticle loads include "super-Gaussians" (jmg $\geq+3$ ) and hard edge, uniformly-filled ellipsoids (i.e. waterbag) in 4-D phase space (jmg < 0 ) (which leads to parabolic radial density profile). If one is interested in accurately diagnosing bunching at higher harmonics, the number of macroparticles needed for good statistics will increase (e.g. $\geq 16384$ for the 7th harmonic).

\subsubsection{Electron Beam Emittance and Size}

By default, the ratio of the $x, y$ and $x^{\prime}, y^{\prime}$ axes of the transverse emittance ellipsoid are chosen such that the electron beam will be in a matched equilibrium (i.e. to prevent downstream mismatch oscillations) with the focusing properties of the wiggler at entrance. For equilibrium loads, the ebeam size is normally determined by input of the normalized MKS emittance (emit_mks in rad-m) or normalized CGS emittance (emito in rad-cm). By default, the emittance is presumed the same in both transverse planes but a new feature permits one to specify different values for the MKS $x-x^{\prime}$ (emitx_mks) and $y-y^{\prime}$ (emity mks) projected emittances. If none of the emittance variables is input, the code calculates the equivalent equilibrium emittance value if the namelist contains either (a) the beam radius (omgj) in $\mathrm{cm}$; or (b) the beam current AND the central beam brightness (bright). For historical reasons, the brightness is defined in "old LLNL" units of Amps/(rad$\mathrm{cm})^{2}$ with $J \equiv 2 I /\left(\gamma \varepsilon_{o}\right)^{2}$ for a uniformly-filled ellipsoid; note the absence of a $\pi^{2}$ factor in the denominator.

GINGER also has a seldom-used capability which determines the beam current when just upstream of the wiggler the electron beam passes through an emittance filter - such a situation was true for the 1980's LLNL/LBNL ELF experiment. To do this, the user must input a negative value for the beam current, a positive value for the electron beam brightness, and a positive value for either the electron beam emittance or radius.

In cases where the focusing strength is different in the $x$-plane from that in the $y$-plane, the equilibrium beam radius in each plane will differ if either the emittance or brightness is specified. 
One may also use the multiplicative factors xbscale and ybscale to set the beam radius in either plane to a larger or smaller value than that corresponding to equilibrium. For uniform ellipsoid loads, the input emittance is the hard edge value; the corresponding RMS value is smaller by $\sqrt{6}$. For Gaussian loads, the input emittance corresponds to the RMS value in each projected plane (i.e. $x-x^{\prime}$ or $y-y^{\prime}$ ), not the edge value.

There are at least two ways in which the user can force the beam radius in each plane to particular values. The first is by giving the Twiss parameter beta in both planes: betax_twiss for $x$ and betay_twiss for $y$, both in meters (note that this is an exception to the "centimeter" units rule for transverse quantities!). Alternatively, one may set the radius in a given plane by inputting the parameters omg jx and/or omg jy, both in units of $\mathrm{cm}$. This feature is particularly useful when there is no focusing in the wiggle plane of a linear wiggler. Input of the Twiss beta parameters overrides any values specified for omg jx and/or omgjy. When specifying the Twiss parameters, one must also specify the emittance. Note: if in one transverse plane there is neither wiggler nor external focusing (as might occur for a linear wiggler without curved pole face focusing — see $§ 3.5 .5$ ), it is highly advisable for the user to input the initial beam size in that plane manually.

The parameter rmaxcur sets the electron beam's cutoff radius (in $\mathrm{cm}$ ) when a Gaussian distribution has been chosen. The default value for rmaxcur is $3 * o m g j$. At present, as rmaxcur/omgj approaches 2 or smaller, the resultant particle load will result in an RMS emittance significantly smaller than input.

\subsubsection{Initial Tilts and/or Offsets in $x, x^{\prime}, y, y^{\prime}$}

By default, there is no tilt of the initial emittance ellipse; i.e. the averages of $\left\langle x x^{\prime}\right\rangle$ and $\left\langle y y^{\prime}\right\rangle$ are zero. One can override this with the Twiss parameters alphax_twiss and alphay_twiss. One may also input the "thin lens" parameters xfocus_mtr and yfocus_mtr which set a hypothetical, zero emittance focal point (in meters) in the $\mathrm{x}$ - and y-planes respectively. For an individual macroparticle " $n$ ", this adds a term $-p_{z} * x_{n} /$ xfocus_mtr and $-p_{z} * y_{n} /$ y focus_mtr, respectively, to the particle's transverse momenta. The net effect of specifying both the Twiss alpha parameters and the thin lens lengths are additive - i.e. one does not override the other. One may also add either a constant transverse offset (xoff and yoff in $\mathrm{cm}$ ) or transverse angle (xprime and yprime in radians) to the electron beam centroid. However, one should remember that for non-waveguide runs GINGER presumes axisymmetric radiation fields and the coupling between an off-axis electron beam and the radiation will not be treated in a self-consistent manner.

\subsubsection{Instantaneous Energy Distributions and Chirping}

The default instantaneous beam energy distribution is a delta function centered at the input-specified e-beam Lorentz factor gammar0 or, alternatively, the beam energy in $\mathrm{MeV}$ (energy). One may 
specify a non-zero energy spread by inputting values for the width dgamma and the distribution type gamload. Permitted values for gamload are (1) ' uniform', the default; (2) 'random' ' or (3) 'gaussian'. Note that these choices are all lower case. For a gaussian distribution, dgamma is the RMS width while for the uniform and random loads, macroparticles are initialized between gammar $0 \pm$ dgamma.

In time-dependent mode, one may also place a chirp on $\gamma(t)$ with an amplitude of gamchirp. If chirp_type is set to its default value of 'sinusoid', $\gamma$ varies sinusoidally with a peak-topeak amplitude of $2 *$ gamchirp. When chirp_type is 'linear', $\gamma$ increases from a value gammar0 at the beam tail to a value (gammar0 + gamchirp) at the beam head.

\subsubsection{Pre-existing, Externally-produced Macroparticle Distributions}

GINGER now has some capability to read in externally, previously generated macroparticle distributions. The input variable trackparfile should be set to the name of an ASCII file which contains the phase space information. At present (November 2001) the format should be arranged as follows:

(1) a number of comment lines with the last one containing the string ' ---------------' ;

(2) the next line should contain the integer number of macroparticles (NP) in the file;

(3) NP single lines, each containing $\left(x, x^{\prime}, y, y^{\prime}, \gamma\right)$ for a single macroparticle, with $x$ and $y$ in meters, $x^{\prime}$ and $y^{\prime}$ in radians, and $\gamma$ being the Lorentz factor of each macroparticle.

GINGER will replicate this external distribution in $\left(x, x^{\prime}, y, y^{\prime}, \gamma\right)$ nfold_sym times (which has a default value of 8 for "quiet start" loads - see §3.3.6) in longitudinal phase space. Thus, if the external distribution file contains 512 distinct particles, each electron beam slice in GINGER will then contain 4096 macroparticles if $\mathbf{n f o l d}$ ssym is left at 8 . For polychromatic runs, each of the nside electron beam slices will have identical distributions in $\left(x, x^{\prime}, y, y^{\prime}, \gamma\right)$. Thus, the only initial microbunching occurs through the application of shot noise effects upon the longitudinal phase $\theta$.

\subsection{6 "Quiet" Starts and Shot Noise}

By default (lquiet=.t), the macroparticles are loaded in phase space with a bit-reversed quiet start with a $N$-fold symmetry in the longitudinal coordinate $\theta$ where $N$ is set by the input variable nfold_sym with a default value of 8 . Thus, each macroparticle at $\left(x_{n}, x_{n}^{\prime}, y_{n}, y_{n}^{\prime}, \gamma_{n}, \theta_{n}\right)$ will have nfold_sym-1 "shadow" macroparticles with the identical $\left(x_{n}, x_{n}^{\prime}, y_{n}, y_{n}^{\prime}, \gamma_{n}\right)$, but whose longitudinal phase $\theta$ is successively incremented by $2 \pi /$ nfold_sym. Choosing nfold_sym $=8$ will eliminate any initial bunching through the fourth harmonic. In studies of third harmonic bunching, we found it necessary to use seven rather than one shadow particle in order to cancel out all initial bunching at the fourth harmonic (which couples to growth of the third harmonic in the exponential gain regime). If one is examining bunching through harmonic $N$, one should set 
nfold_sym $=2 \mathbf{N}+2$ and simultaneously increase ntestp appropriately to retain reasonable resolution in $\gamma$ and transverse phase space quantities. However, if one is not concerned with accurate modeling of higher harmonic bunching, picking nfold_sym=2 will give the best resolution of the transverse phase space and longitudinal energy spread distributions for a given value of ntestp.

When shot noise fluctuations are desired (lshot=.t) for SASE and similar studies, a random $\delta \theta_{n}$, which follows a Poisson distribution, is added to each macroparticle's longitudinal phase $\theta_{n}$. There are no fluctuations in transverse phase space. In fall 1999, the shot noise algorithm was completely rewritten to try to overcome a small bug observed when dgamma was non-zero. Now, each group of nfold_sym macroparticles with the same $\left(x_{n}, x_{n}^{\prime}, y_{n}, y_{n}^{\prime}, \gamma_{n}\right)$ has its own set of random shot noise variables (e.g. bunching phase and amplitude at different harmonics of the fundamental radiation wavelength $\lambda_{s}$ ). The new algorithm appears to ensure that coarse-grained averages over both $<\exp i \theta>$ and $<\exp 3 i \theta>$ are correct (and have identical values in the limit nside $\rightarrow \infty$ ). One may increase/decrease the effective power level of initial shot noise bunching fluctuations by setting pwrnoise different from its default value of one. We have found that comparisons of computed growth from shot noise in both low and high gain FEL's with that expected analytically have provided an excellent debugging tool for GINGER.

Shot noise bunching fluctuations may also be included in monochromatic, single-slice FREDmode runs. This capability is useful if one wants to quickly examine the differences between MOPA's and SASE-like input sources. However, one should remember that, given the absence of slippage in FRED-mode runs, these fluctuations will be "coherent" (and thus monochromatic) in a longitudinal sense and will also produce a much larger effective input laser power than would be true for the equivalent polychromatic run.

\subsubsection{Random Number Seeds}

Random number seed input variables (which, in principle at least, should allow the user to repeat exactly previous simulation runs) include: (1) iseeds for longitudinal shot noise (see $\S 3.3 .6$ ); (2) iseed for the phase and amplitude of the different radiation field spectral components (see $\S 3.4 .3$ ); and (3) iseedp for loading instantaneous energy spread (applicable when gam_load=' random' ; see $\S 3.3 .4)$. Due to recent changes in the shot noise algorithm, the variable iseedp no longer affects the electron beam's transverse phase space distribution.

Each of the random seed variables is a 4-element array of decimal (i.e. not octal) integers. A "master seed" is created by a call to the system clock which is then used to generate those seed variables not input by the user. In this case, the generated seed will lead to only the first element of the seed array being non-zero. However, if the user inputs a random seed variable (as might be true to recreate a run), this variable is converted into an array of 4 12-bit integers (i.e. the effective seed is the input seed modulo $2 * * 48$ ). Hence, one should not input a seed greater than $\sim 2.8 \times 10^{14}$. For runs on the NERSC CRAYs (which have 64-bit size words), the first array input seed element can 
fully contain the 48-bit effective seed. On platforms with 32-bit integers (including the IBM-SP and most workstations) one would need two array elements to get the full dynamic range of possible input seeds (however, it is not likely any user will do such a large number of runs that $2 * * 32$ unique seeds proves insufficient!).

For all runs (FRED-mode and polychromatic-mode, single and multiprocessor platforms) with GINGER versions beginning in November 2001, the random numbers have effective lengths of 48-bits and are generated by a special numerical package provided by NERSC consultants. For multiprocessor runs, this package has the distinct and needed property that the random numbers used to generate each beam slice will be independent of both the total processor number used in the run $A N D$ the particular assignment order of individual processors to individual slices.

\subsubsection{Longitudinal Current Profiles}

By default, the electron beam current is time-independent (pulse_shape=' tophat' ). For short pulse, polychromatic simulations with either ltransit=.t or losc $=. t$ which override periodic boundary conditions in time, other longitudinal current profile options include (a) parabolic (pulse_shape=' parabolic'), (b) Gaussian (pulse_shape=' gaussian'), or (c) flat-top with an exponential rise and fall (pulse_shape $={ }^{\prime}$ exptail'). For parabolic profiles, the full pulse width will be equal to (nside/nsidep) $\times \tau_{\text {slip }}=($ nside/nphoton $) \times$ window. For Gaussian profiles, the input variable tbody sets the RMS pulse width in seconds. One must be careful that tbody is appropriately small enough for the choice of nside and window. For flat-top profiles, trise sets the exponential rise and fall time in seconds.

GINGER does not presently model pre-existent coherent microbunching on the electron beam at its entrance into the wiggler. Such bunching (which produces coherent spontaneous emission) can be important for electron beams whose pulse lengths are tens of radiation wavelengths or shorter, especially when the longitudinal profile is non-Gaussian. Since this coherent microbunching can in principle be much larger in magnitude than the incoherent shot noise term, its absence is an important limitation in GINGER.

\subsection{Radiation Field Input Quantities}

\subsubsection{Optical Beam Size, Profile, and Waist Position}

Paralleling many of the electron beam input variables are those corresponding to the radiation field. The default initial transverse profile is Gaussian $(\mathbf{n m g}=+2)$ while "supergaussians" may be specified by $\mathrm{nmg} \geq+3$. The optical waist radius $\omega_{0}$ (三omg0) corresponds to the $1 / e$ point in $\vec{r}$ of the radiation electric field when at a waist minimum. Normally, omg 0 is determined by the input variable omg 0 fac, whose default value is 0.8 , and the relation omg $0 \equiv$ omg $j \times$ omg 0 fac, where 
omgj is the electron beam radius. This can be overridden by giving a positive value for omg 0 , Optionally, one may also set the position in $z$ of the focal point (i.e. waist) of the input radiation by giving a value either in meters (zfcmeter) or in Rayleigh ranges (zfocus). The Rayleigh range $Z_{r} \equiv \pi \omega_{o}^{2} / \lambda_{s}$. A non-zero value for either leads to curved wavefronts at $z=0$. Note that although the electron beam model includes full 3-D non-axisymmetric dynamics which can result in a non-circular shape, the radiation field and its source terms are presumed axisymmetric (in nonwaveguide runs).

\subsubsection{Wavelength, Input Power, Slice \#, and Temporal Resolution}

By default, GINGER presumes a time-dependent, polychromatic problem; this can be overridden by setting either 1 fred $=. t$ or $n s i d e=1$.

The optical wavelength $\lambda_{s}$ is specified by wavels in meters; this is the numerical value of the central wavelength of the effective bandpass in polychromatic runs. If one is running a microwave problem (lwavegd=.t), one may alternatively specify the central frequency in $\mathrm{GHz}(\mathrm{ghz})$. Normally, one uses the input variable plaser to set the initial radiation power in watts.

There are a number of input parameters which define the temporal resolution of the input laser field. The variable nphoton sets the total number of photon slices. For short-pulse, non-periodic boundary conditions (e.g. oscillator or "transit time" runs), nphoton must be input. For periodic boundary conditions in time (e.g. long pulse amplifier runs), nphoton = nside automatically and nside rather than nphoton should be input. In any case, the resultant nphoton should be a power of two or three times a power of two in order for the FFT spectral decomposition in the postprocessor to run properly. To set the total temporal duration of the radiation field and the time interval between individual photon (and electron beam) slices, one should set either window, which gives the equivalent longitudinal length (in meters) of the temporal window, or nsidep which gives the total number of discrete photon slices with which a particular electron beam slice will interact over the full length of the wiggler. Numerically, window $\equiv($ nside / nsidep $) \times\left(L_{w} \times \lambda_{s} / \lambda_{w}\right)$. When nsidep $=N_{w}$, the full frequency span of the simulation equals central frequency $\left(\equiv c / \lambda_{s}\right)$. In the great majority of situations one will usually set nsidep rather than window.

At present with one exception, nsidep must be less or equal to nphoton. The exception exists for the multiple processor runs on MPP platforms (the CRAY-T3E and IBM-SP) where, nsidep can exceed nside. In cases where the slippage length greatly exceeds the so-called "cooperation" or "coherence" length, one should consider employing this strategy to increase the $z$-resolution and spectral bandpass without having to increase the total number of slices. This option is also useful for optical klystron configurations (§3.7.2). 


\subsection{3 “Customizing” Time-dependent, Input Radiation Fields}

The user has a fair amount of flexibility in "customizing" the slow time dependence of the input laser field. If, for whatever reason, one wants the wavelength of the input radiation field (to which plaser refers) to be different from the central wavelength of the simulation (=wavels), one can set this by wavelsin in meters. If one wants the radiation power spread equally over a number of spectral bins, one gives a positive integer value for nfreqbin. Both wavelsin and nfregbin may be simultaneously input.

To generate a uniform spectrum with equal noise power in each of the nphoton frequency bins encompassing the complete frequency span, one sets the power level per bin by either inputting wattpbin or wattpghz. The latter applies only to waveguide runs. If plaser $=0$., there will be only noise (including at the central frequency) resulting in a total input power of nphoton $\times$ wattpbin. Another means of starting with a uniform power spectrum without excess power at the central frequency is by giving a positive value for plaser and a negative value for nfreqbin in which case the total input power is plaser exactly. Alternatively, a negative value of ampside together with a positive value of plaser also generate a flat noise spectrum over the full bandpass (in addition to the power plaser at the central wavelength) with the total noise power equaling (plaser $\times$ ampside ${ }^{2}$ ). An example of this type of white noise initialization is given in $\S 3.12 .2$.

If one wants all the non-fundamental power at a single frequency or wavelength, one specifies either sidewave in meters or sidefreq in $\mathrm{Hz}$. The power level is set by a positive value of ampside with $P_{\text {sideband }}=$ plaser $\times$ ampside ${ }^{2}$. A positive ampside without specifying either sidewave or sidefreq sets up a white noise sideband field amplitude $\hat{E}(r, t)$ fluctuating in time between \pm ampside $\times E_{o}(r)$ where $E_{o}(r)$ is the electric field of the fundamental.

When running in short-pulse mode (i.e. Itransit $=. t)$, the radiation field is not presumed to be periodic in time and for various reasons GINGER does not initialize all nphoton locations in time of the radiation field at $z=0$ (essentially it needs only to initialize those radiation slices which immediately interact with the electron beam). Consequently, even if one specifies a flat noise spectrum (e.g. via the wattpbin input variable), the post-processor power spectrum will not show a flat spectrum at $z=0$.

Finally, one may place a sinusoidal temporal chirp on the input radiation's wavelength by giving a positive value to the input variable ampchirp, which defines the peak normalized (i.e. relative to the simulation bandpass) wavelength shift. In general, one would use such a chirp only for "long pulse" simulations with temporally-periodic boundary conditions. 


\subsubsection{Saving to and Initializing from $z$-dependent Radiation Field Files}

A new, still-evolving feature in GINGER allows a user to save to a special disk file the instantaneous state of the radiation field "exiting" from the "head" e-beam slice (i.e. slice \#nside as GINGER numbers slices beginning from the tail). Because GINGER applies slippage at nsidep discrete locations in the wiggler (see §5.5), this "exiting" field information is written at the same $z$ - locations. This field information can subsequently be used at the beginning of a new GINGER run to initialize the radiation field for the new "tail" e-beam slice (i.e. slice \#1). This procedure results in the equivalent of stitching together of two temporally-adjoining electron beam sections; i.e. it is only through such radiation slices that adjoining electron beam slices "communicate".

There are two underlying purposes for this feature: (1) To permit a user cut up a relatively long electron beam pulse (as compared with the slippage length) into a number of shorter sections, each one of which can then be run sequentially with GINGER. In effect, each run following the first is similar to a short pulse, transit-time run but with no radiation field propagation into vacuum beyond the electron beam body. (2) To allow a user to do a monochromatic, single-slice "FRED-mode" run using the radiation field from a polychromatic, time-dependent run. The macroparticle output diagnostics from such a "FRED-mode" run (via the nspec input variable) can be used to examine in great detail the $z$-evolution of an electron beam slice's longitudinal $\gamma-\theta$ phase space.

To save a $z$-dependent field file, set the input variable 1 _write_fld_z $\mathbf{z}$. in the main namelist. This "write"switch may be used in "long-pulse", periodic BC polychromatic mode only (losc and Itransit are both false) for both single- and multi-processor runs. At the completion of the simulation, an ASCII SDDS-formatted file named fldrun_name.sdds (e.g. fldpalac.sdds) is written to disk. In addition to the complex radiation field information, the numerical values of the radial grid are also stored (see $\S 3.9 .1$ ).

To use such a pre-existing $z$-dependent field file in a subsequent run, set 1 _read_fld_z $=. t$ in the main namelist. Also, the execute line of GINGER must now include the option $-f$ fldfile, where fldfile is the full name of the previously created SDDS-format field-file. The new run can be either in single slice, FRED-mode or multiple slice, polychromatic-mode. In either case, the electron beam and wiggler parameters of the new run should be absolutely identical to those of the previous one which created the field-file (future GINGER work may allow a slow time variation of e-beam parameters in this mode, similar to what exists already in true short-pulse mode). In polychromatic mode, the new run should have the same value of nsidep but nside can now

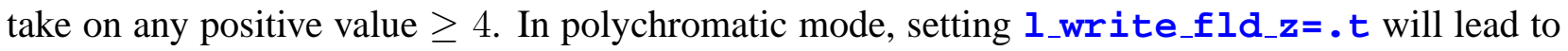
the writing of a completely new field file; be careful not to use the same run_name as that of the previous run or the old field file will get overwritten. In FRED mode, the input variable ncurve must be set to the previous run's value of nsidep.

The post-processor XPLOTGIN also now has capabilities to generate a $z$-dependent fieldfile from the field information contained in the pltfile of a previous run (see $\S 4.11$ for details). 


\subsection{Wiggler and Focusing Input Variables}

\subsubsection{Base Wiggler Input Parameters}

Nearly all GINGER runs require the user to set the following input variables to specify the wiggler: polarization type with llinear $=. t$ for a linear wiggler or llinear $=. \mathbf{f}$ for a helical one, wiggle period wavelw in meters, and whether the wiggler field strength is constant with $z$ (lcnstwgl=.t) or not (lcnstwgl=.f). The total wiggler length (including drift spaces, if any) is set equal to the simulation's longitudinal span in either Rayleigh ranges (zmaxsim) or meters (zmxmeter). Beginning in November 2001, an optional "lattice" file may be used to set wiggler parameters (including pole strength errors and corrective steering) — see $\$ 3.5 .8$ for details.

\subsubsection{Constant $a_{w}(z)$ Wiggler Field}

The longitudinal dependence of the RMS normalized vector potential $a_{w}(z)$ can be set in several ways. A constant strength wiggler can be specified by giving one of the following: (1) a positive value for aw0, the $R M S$ normalized vector potential strength of the wiggler on axis; (2) a positive value for bw0, the peak, on-axis wiggler field strength in Tesla (note: changed from $\mathrm{kG}$ as of Nov 2001); (3) setting idesign=1, following which GINGER computes the appropriate resonant value for $a_{w}$ corresponding to the input values for gammar0, wavelw, and wavels. In the last case, no allowance is made for detuning effects such as non-zero emittance and/or quadrupole focusing which normally will make peak gain occur at a slightly lower value of $a_{w}$. One can scale the nominally computed $a_{w}$ up or down by giving a value for the input parameter awdmult different from its default value of 1.0 .

\subsubsection{Using a Predetermined Tapered Wiggler $a_{w}(z)$ Profile}

In some cases one may want to use a predetermined tapered wiggler stored in a separate ASCII file. To do so, (a) set idesign=0; (b) set lenstwgl=.f; and (c) specify the name of the wiggler file by using the $-\mathrm{b} b$ wfile option on the execute line. The previously mentioned input parameter awdmult can also be used to scale an input tapered $a_{w}(z)$.

Normally, such a tapered wiggler file will have been generated by a previous GINGER run operating in FRED-mode. However, the user can also design such a file following this format:

(1) the first line is simple text. Normally this contains some details about the GINGER run which generated it but the user may put anything here.

(2) The second line contains a single integer equal to the number (三N_AW) of $z$ positions to follow; a minimum of two such positions is needed.

(3) $\mathbf{N} \_$AW single lines containing two floating point variables: the $z$ position (in meters) and the on-axis value for $a_{w}(z)$. 
By default, GINGER will use a cubic spline fit to determine the local $a_{w}(z)$ from the discrete values of $a_{w}$ input in the wiggler file. If one desires abrupt shifts in either $a_{w}$ or its $z$-derivative, this can require putting in quite a few additional points to force such a shift in the fit. However, one can avoid this annoyance by setting the input namelist variable $1 \_$bw_linearfit $=. t$ which replaces the spline fit with simple linear interpolation of $a_{w}$ between adjacent points in $z$.

\subsubsection{Tapered Wiggler Self-Design in FRED-Mode}

In FRED-mode only, setting idesign=2 and lenstwgl=. $\mathbf{f}$ uses GINGER's self-design algorithm to compute a tapered wiggler. These $a_{w}(z)$ values will be written out to the bwfile at ncurve equally spaced locations in $z$. The algorithm determines $a_{w}(z)$ by keeping its ponderomotive well phase $\psi(z)$ constant for an imaginary test particle initially located at $\psi=$ psir 0 , $\gamma=$ gammado, and transversely at $r=r d e s i g n$. Default values are psir $0=0.4$ and gammado $=$ gammar0. The switch 1 phase $10=. t$ limits the $z$-rate of change of $a_{w}(z)$ caused by the electromagnetic field phase variation $d \phi / d z$; normally, this is only a concern for high gain microwave FEL's. In order to account for the radial increase of $a_{w}$ off-axis and the radially local value of the radiation field phase $\phi(r)$, the parameter rdesign or rdesfac determines the radius of the imaginary design particle, where rdesign $=$ rdesfac $\times$ omgj. with a default values rdes$f a c=0.707$. A sample tapered wiggler input file is given in $\S 3.12 .1$.

\subsubsection{Wiggler Focusing: Simple and Curved Poleface}

Wiggler focusing is controlled by a number of optional input parameters. Helical wigglers have natural focusing in both transverse planes while linear wigglers have focusing only in the nonwiggle plane ( $\hat{y}$-direction in GINGER for non-waveguide FEL's). If no other focusing than the "simplest", natural wiggler focusing is desired, no additional input parameters are required.

However, for longer wigglers with linear polarization, one generally needs focusing in the nonwiggle plane. One method to achieve this is by using "curved" (aka "parabolic") pole piece focusing by inputting a value for $\mathbf{r k x k w}$ which increases the focusing in the wiggle $\hat{x}$ plane while simultaneously reducing the focusing in the non-wiggle $\hat{y}$ plane. rkxkw must lie within the interval $[0,1]$.

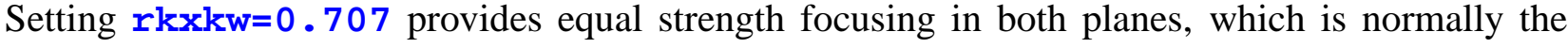
desired result.

\subsubsection{External Focusing: Continuous Quadrupoles and/or Ion Channels}

If one desires external focusing is addition to that provided naturally by the wiggler, GINGER provides a number of choices: 
(1) To add constant, $z$-independent, quadrupole focusing in the wiggle plane (and simultaneously equal magnitude defocusing in the non-wiggle plane), specify a positive value for quado, which has units of Gauss/cm, or wavelx, the desired betatron wavelength $(\mathrm{m})$ in the wiggle plane. Alternatively, a positive value for focusfac, which measures the ratio of the quad-induced $k_{\beta, x}^{2}$ relative to the value of $k_{\beta, y}^{2}$ associated with the natural wiggler focusing, will also give wiggle plane focusing. If $a_{w}$ varies with $z$ (i.e. a tapered wiggler), so will the actual quad gradient when focusfac is non-zero. When focusfac $=0.5$, the net focusing (i.e. quadrupolar plus wiggler) will be equal in the wiggle and non-wiggle planes. In general, one will input quado, focusfac or rkxkw only for linearly-polarized wigglers.

(2) One may add "strong" and equal focusing in both planes by setting zlion equal to the wanted (external) betatron wavelength in meters for a particle at $\gamma=$ gammar 0 . This is a "kluge" to simulate the long-wavelength effects of AG quadrupole motion but ignores the short wavelength AG flutter. zlion also simulates the effects of a focusing ion channel (hence the name).

\subsubsection{External Focusing: Discrete Quadrupole Magnet Lattices}

GINGER now can include discrete quadrupole magnets in a periodic lattice, which may range from a simple FODO system to more complicated systems such as triplets. At present, only one periodic lattice can be defined but each lattice cell may include up to ten individual quadrupoles, each of which can have its own length and gradient. The necessary input variables are:

(1) quad_lattice_zperiod, the full lattice period (m)

(2) quad_lattice_zstart, the beginning position (m) of the first lattice cell

(3) quad_lattice_zend, the end position (m) of the last lattice cell (the default value of $10^{6} \mathrm{~m}$ exceeds any reasonable wiggler length)

(4) quad_lattice_mag_gradient, an array containing the individual magnet gradients in G/cm. A positive gradient corresponds to focusing in the $x$-plane

(5) quad_lattice_mag_start and quad_lattice_mag_zend, arrays defining the beginning and end $(\mathrm{m})$ of the individual quadrupoles relative to the beginning of each periodic lattice cell

(6) quad_lattice_nmag, the number of magnets in each cell. This is an optional parameter which need not be input because GINGER uses the input gradient values to determine how many magnet truly exist in the lattice.

To make sure that each individual magnet will be "hit" at least once by GINGER's PDE integrator, the maximum step size hub $m$ in meters should be set to approximately $75 \%$ or less of the shortest quadrupole's length (see $\S 3.9$ for information on numerical integrator parameters). Also, individual magnets should not overlap one another. At present, all magnets have "hard" edges and no fringe field effects are calculated. 


\subsubsection{Lattice Files, Wiggler and Quadrupole Errors, and Steering Corrections}

As of November 2001, GINGER now has the capability for linearly-polarized undulators (only) to model the transport effects of pole excitation errors, quadrupole offset and gradient errors, and dipole steering corrections. As the treatment of these new features matures, they may evolve in various ways including input format, physics description, etc. Currently, to input such errors and steering corrections (if any) and model their effects, the user should set the main namelist input variable lattice_file equal to the name of special ASCII lattice file. This file (which is normally produced by an external program XWIGERR; see $\S 3.5 .9$ below) has the following format:

(1) a single line containing the string "GFLD", starting in the first column

(2) an arbitrary number of comment lines with the last one signified by the string " $\$$ " in columns 2 and 3

(3) a Fortran90 namelist "lattice" which should contain the following variables: gammar0, wavelw, aw0 (these values should agree exactly without those input in the main GINGER namelist), nelement (the total number of discrete sections, including drifts, which describe the undulator) and the variables nquad and nsteer which define the number of individual quadrupoles and steering elements to be described.

After the namelist, the sets of individual quadrupoles, steering dipoles, and wiggler elements are read (in that exact order). The individual quad format is the string "QUAD" in the first 10 characters, followed by five real numbers giving the beginning and end z-position in meters, the quad gradient in $\mathrm{T} / \mathrm{m}$, and the $\mathrm{x}$ - and $\mathrm{y}$-offsets in meters. The steering element format is "DIPOLE" in the first 10 characters, followed by 3 real numbers giving the $\mathrm{z}$-position in meters and the $\mathrm{x}$ - and $\mathrm{y}$ integrated fields in T-m. The wiggler element format is either "WIGGLER" or "DRIFT" in the first 10 characters, followed by four real numbers. For the case of "WIGGLER", these numbers comprise the initial z-position in meters, the nominal value of aw0 (without errors), the pure "phase error" component of aw0, and the effective "kick error" component of aw0 (which is acts as a transverse dipole magnetic field in the wiggle plane). These error values are usually specially-averaged values over multiple wiggle periods (see $\$ 3.5 .9$ ). For the "DRIFT" case", only the first two numbers are used which give the beginning and end $\mathrm{z}$ positions in meters of the drift.

Note that the configuration described in the lattice_file, including drift space and quadrupole lattice information if present, override any values set in the main GINGER input file. Consequently, to avoid inadvertent confusion, the user is advised that when modeling wiggler errors, the lattice_file should serve as the primary source of undulator description.

\subsubsection{Generation of Lattice File via the XWIGERR Program}

A simple F90 program (XWIGERR), freely available from the author, generates for a linearlypolarized, constant $a_{w}$ wiggler the individual undulator pole errors and quadrupole gradient and 
offset errors (if any), computes the appropriately-averaged (see below) errors in phase and drift, the proper transverse steering (if any) including the optional effects of beam position monitor offset errors, and then writes a properly-formatted lattice_file (see \$3.5.8) to be used by GINGER.

XWIGERR uses a simple F90 namelist which uses as much as possible the same input variable names as GINGER for equivalent items. Note: units are MKS for both longitudinal and transverse variables, which leads to some inconsistency with GINGER for items such as xoff. Electron beam properties include gammar0 for Lorentz factor (alternatively energy in $\mathrm{MeV}$ may be given), xoff and yoff in meters for initial beam offset, and xprime and yprime in radians for initial beam angle. Main undulator variables are wavelw (or period) for undulator period, zmxmeter for wiggler length, rkxkw to set the amount of curved poleface focusing, and aw0 for RMS normalized vector potential. Undulator error variables include aw_rms_err for RMS fractional $a_{w}$ error, iseed_aw for the optional random number seed for these errors, and nw_avg which is the number of undulator periods over which to specially average the pole strength errors (default value 1). One should not average over a length greater than about one-eighth the effective betatron period $\lambda_{\beta}$ of the system. Drift sections are described using the same variables as those in the lattice formulation of the main GINGER namelist (see §3.7.1).

If there are discrete external quadrupoles, the lattice may be described by the exact same variables as in the main GINGER namelist (e.g. quad_lattice_zperiod; see §3.5.7). Additional quad input variables include quad_lattice_rms_offset for the RMS offset in meters, quad_lattice_rms_grad_err for the RMS fractional error in quadrupole gradient, and mag_units which is either ${ }^{\prime} T / \mathbf{m}^{\prime}$ (default) or ${ }^{\prime} G / \mathrm{cm}^{\prime}$. The optional random number seed iseed_quad can be used to generate the offset and gradient errors.

If discrete dipole steering correction kicks are wanted, the following namelist variables apply: zsteer_beg_end for z-interval in which steerer are placed (default full wiggler length), zsteer period for the period interval of steerers, and zsteer 0 which gives location of an optional steerer before the beginning of the periodic lattice - if used, this is normally placed at the beginning of the wiggler. In place of these three variables, the user may instead input discrete steerer $z$-locations via the real array zsteer. One may also input RMS beam position monitor transverse errors via bpm_rms_offset in meters and a random number seed iseed_bpm for such. Presently, the steerers are presumed to have infinitesimal longitudinal length and GINGER applies them as delta-function transverse kicks.

The XWIGERR namelist variables outfile set the main output filename ( $\equiv$ the GINGER input lattice_file), sdds_outfile the SDDS-formatted filename which contains the predicted beam $x$ - and $y$-centroid versus $z$ (including effects of steering), and description an $\leq 80$-character string may be used to set a comment in the outfile. 


\subsubsection{Checking Beam Transport Properties through the Lattice}

In order to check the beam transport (i.e. beam envelope size and centroid motion) through the wiggler's focusing lattice, it may be useful to do a single slice, FRED-mode run with the input parameter nspec, which controls the number of phase space dumps, being set to a moderate positive value. Then using the 1 plot $\mathbf{x y}=$. $t$ switch in the XPLOTGIN preferences file (see $\S 4.12$ ) forces the postprocessor to produce $x-y$ macroparticle scatter plots at various z-locations. History plots of envelope variables $\sigma_{x}, \sigma_{y},\langle x\rangle$, and $\langle y\rangle$ versus $z$ are also automatically produced in single slice FRED-mode runs. To speed up this sort of debugging run, one can set lfixfld=.t to prevent any $z$-evolution of the radiation field, and luncoupl $=. t$ to decouple the macroparticles's $z$-evolution in $\gamma$ and $\theta$ from the radiation field. Also, the switch lmovxyt should be left at its default value of .true. in order to retain macroparticle betatron motion.

For debugging and investigatory purposes, GINGER has a number of other logical switches which control details of the physics included in the macroparticle motion. When lawaxis $=. t$, the radial variation in $a_{w}$ is ignored in the KMR equation for longitudinal phase advance. Using this switch leads to an unphysical variation in $\gamma_{\|}$over the betatron orbits of individual macroparticles. The switch lmovxyt $=$. $\mathbf{f}$ prevents all macroparticle betatron motion. The switch lxvxfix=.t freezes motion only in the $\hat{x}$ plane and was implemented for TE0n mode waveguide studies.

\subsection{Waveguide, Space-charge, and External $E_{z}$ Input Parameters}

\subsubsection{Specification of Waveguide Properties}

By setting lwavegd=. $t$, one instructs GINGER to propagate the radiation within a rectangular waveguide, in which case the radiation's longitudinal wavenumber $k_{z} \neq \omega_{o} / c$. The waveguide dimensions are specified via xwidth and ywidth, both in $\mathrm{cm}$. Rather than using radial gridding as is true for non-waveguide simulations, in waveguide mode GINGER adopts a Cartesian grid in the $y$ plane and, by default (1te21=.f), follows the TE01, TE03, TE05, ... modes. For these modes, the electromagnetic field is independent of the wiggle plane direction $\hat{x}$, the (normally) larger waveguide dimension (i.e. $x w i d t h \geq y w i d t h$ ). The code does not actually decompose the field into the various TE modes; their strength is diagnosed only in the postprocessor through analysis of the spatial variation of the field on the grid. Note that when doing polychromatic runs, there is no variation of group velocity with $\omega$ or $k_{\perp}$; in other words, the slippage velocity is independent of $\omega$.

When 1 te21 =.t, the code follows the TE01, TE21, TE41, .. modes and, somewhat confusingly, one must set the waveguide "on its side" by making the long dimension in the $y$ direction (this is required because of the internal gridding in $y$ ). In this latter case, the TE41 mode will follow a $\cos (\pi x / \mathrm{xwidth}) \times \cos (4 \pi y / \mathrm{ywidth})$ spatial dependence with the coordinate origin lying at the center of the waveguide. Note: if one desires to set a positive quadrupole focusing in the wiggle 
plane, one still uses a positive value for wavelx or quado even when the wiggle plane is parallel to $y$ for 1 te21=.t. This is also true for other focusing parameters such as rkxkw, and focusfac. Internally, the code computes $k_{\beta}$ with the wiggle plane parallel to $x$ and then exchanges the $x$ and $y$ directions.

Since most microwave FEL's operate at relatively low energies, the approximation $\beta_{\|} \approx 1-$ $1 / 2 \gamma_{\|}^{2}$, which GINGER normally uses, can lead to inaccuracies. These can be prevented by setting the input variable lbetapar $=. t$, which forces exact evaluation of $\beta_{\|}$in the equation determining the macroparticle longitudinal phase derivative $d \theta / d z$.

\subsubsection{Longitudinal Space-charge}

When modeling high current microwave FEL's such as the LLNL/LBNL ELF experiment, longitudinal space charge forces can become important and affect both particle microbunching and radiation field gain. If Ispacech=.t, GINGER follows these forces using the same approximations as did the original FRED code; the interested user can find physics details in the paper by E. T. Scharlemann et al., Nucl. Instr. Meth. Phs. Res. A250, 150 (1986). The space charge switch also works for non-waveguide FEL's. Inclusion of space charge forces will increase running times by a factor of two or more for most problems. The effects of transverse space charge fields are not modeled by GINGER; their net force relative to the "pressure" term corresponding to the electron beam transverse emittance is usually very small due to cancellation by the electron beam's azimuthal magnetic field.

\subsubsection{External Accelerating/Decelerating Fields}

For various reasons, the user may want to examine the effects of a superimposed uniform (in both $r$ and $z$ ) accelerating or decelerating longitudinal electric field or equivalent energy loss term e.g. incoherent synchrotron emission. A gain or loss term is set by the input variable dgamdzo, the change in Lorentz factor $\gamma$ per meter. A positive value accelerates the macroparticles. There is a related switch 1_wakefield=. $t$ which modifies the accelerating field (in polychromatic runs) to be sinusoidal in time with a time period equal to the simulation's periodic window. This latter switch can be used to extract some indication of the resonance-detuning effects of longitudinal wakefields. In general, for e-beam pulses long compared to the "cooperation" length, one would want to be sure that the simulation's time window is also sufficiently long to avoid an unphysical overestimate of such wakefield effects. 


\subsection{Drift Space and Optical Klystron Input Variables}

\subsubsection{Periodic Drift Spaces}

GINGER is capable of including spatially periodic drift spaces in which the wiggler field strength is zero. Such spaces physically might be used for diagnostics and/or discrete quadrupole focusing. At present, only one such periodic drift space lattice can be set but within the period, up to five individual drift spaces may be defined. Four input variables in the main namelist specify the drift space lattice: (1) DL_zperiod specifies the period in meters of the drift space lattice; (2) DL_zstart specifies the point at which the first drift space "lattice" period begins, with a default of the start of the wiggler; (3) DL_zend specifies the point at which the last period of the drift space lattice ends, with a default of the end of the wiggler; and (4) the input array DL_zdrift_beg_end specifies the physical beginning and end in meters of each of the individual drift spaces relative to the beginning of each drift space lattice period.

For example, setting zmxmeter=100., DL_zperiod=10., DL_zstart=25 ., DL_zend=75., and DL_zdrift_beg_end = 3. 5. $8.8 \quad 9.5$ defines a 100-m wiggler containing five drift space 'super-periods' of ten meters each. Within each period, are two individual drift spaces, one 2-m long and the other $0.7-\mathrm{m}$ long. The first 2-m drift space begins at $z=28 \mathrm{~m}$ and the first $0.7-\mathrm{m}$ long drift space begins at $z=33.8 \mathrm{~m}$.

Within the drift space, both the electrons and radiation propagate "normally" but with $a_{w} \equiv 0$ which eliminates any coupling. Hence, there is radiation diffraction but no refraction or gain. In polychromatic mode, GINGER does compute the actual physical slippage occurring in drift sections. In general, this slippage is much smaller than for normal wiggler sections (the opposite from optical klystron sections - see below).

\subsubsection{Optical Klystrons}

The net gain per pass in a low gain oscillator can often be significantly enhanced by the use of an optical klystron dispersive drift section in the wiggler. Such devices have also on occasion been proposed for single pass amplifier configurations to reduce overall wiggler length. GINGER has some ability to simulate optical klystron dispersive sections and, at present, a wiggler may include up to 8 individually unique dispersive sections.

To define their properties, one must set 1 _extra=.t to force the reading of the in_extra namelist. Then, within the in extra namelist, the location of the dispersive sections in meters or Rayleigh ranges are set by giving non-zero values to the arrays $z_{-} O p t K l_{y}$ or t_optKIy, respectively. The physical lengths in meters of the dispersive sections are controlled by the array OptKly_zlen. These lengths define the distance the radiation field propagates with essentially no interaction with the electron beam ( $a_{w} \equiv 0$ as with drift sections), but diffraction does occur. Hence, the effective number of wiggle periods available for gain will be reduced from that of the 
same length wiggler with no drift or klystron sections. The dispersive strengths of the klystron sections are set by the array d_colson. GINGER follows Colson's definition with d_colson defined as the ratio of the dispersion produced in each klystron section divided by that which would be produced by the full wiggler containing only "normal" wiggle periods (i.e. no optical klystron or simple drift sections). Thus if d_colson $(2)=0.2$, the wiggler period is $0.1 \mathrm{~m}$, and the full wiggler length is $10 \mathrm{~m}$, the second dispersive section would produce dispersion equivalent to that normally produced in $0.2 *(10 / 0.1)=20$ wiggler periods.

In polychromatic mode, GINGER presumes that each dispersion section will produce extra physical slippage between the e-beam slice and the radiation field. Numerically, the slippage in each section is set to $\mathrm{d}_{-}$colson $\times \lambda_{s} \times L_{w} / \lambda_{w}$. In reality, the exact value of the slippage will depend upon details of the magnetic design of each optical klystron and might be quite different from this presumed value. Note that when nsidep is given as an input parameter (as is normally done for polychromatic runs), the increased slippage in dispersion sections effectively leads to shortened slippage interaction $z$-intervals in each klystron and lengthened interaction distances in the normal wiggler sections.

For large values of d_colson, one can end up with nearly all the slippage advances occurring in the dispersive sections and very few in normal wiggler sections. This consequently reduces the $z$ resolution of the diagnostic pltfile. To provide partial alleviation for this problem, when running with multiple processors on a MPP platform such as the T3E, one may set nsidep larger than nside this permits greater $z$-resolution. Moreover, as of July 2000, for each individual dispersion section, diagnostic output will be written out at no more than one $z$-location to the pltfile. This change was made to prevent pltfiles from being completely dominated by output from the dispersion sections, where there is little physical change in either the e-beam or radiation.

One should note that only the differential phase advance (relative to a hypothetical resonant particle) in a dispersion section is calculated for each macroparticle; i.e. the physical slippage mentioned in the previous paragraph does not directly affect a macroparticle's longitudinal phase $\theta$. Numerically, $\theta_{\text {out }, n} \equiv \theta_{\text {in }, n}+\left(\gamma_{n}\right.$-gammar 0$) \times 2 \pi \times$ d_colson $\times L_{w} / \lambda_{w}$. The rationale behind decoupling the physical slippage and the dispersive phase slippage was to avoid having the user have to worry about zero order (in $\gamma$ ) phase jumps in $\theta$ as compared with the more important aspects of the energy-dependent phase jump. Nonetheless, there is an option to include an additional energy-independent phase jump by inputting a non-zero value for theta_drift in radians - this term does not lead to additional physical slippage between the radiation and electron beam in timedependent mode. One might use theta_drift to unphysically adjust the net jump in the e-beam longitudinal bunching phase relative to the radiation phase $\phi$, 


\subsection{Oscillator Mode Input Variables}

GINGER currently has a limited capability to model FEL oscillator configurations. Setting losc=.t in either FRED- or polychromatic mode instructs GINGER to model a "short" electron beam (i.e. nside < nphoton and non-periodic boundary conditions in time) with the radiation pulse propagating between two mirrors. In fact, when GINGER (when running on a single processor) models a single-pass amplifier with non-periodic boundary conditions (i.e. Itransit=.t), it secretly forces losc to be true and treats the run as a single-pass oscillator. As mentioned previously, oscillator runs require linking the GINGER executable with the IMSL mathematical subroutine library. Presently, GINGER can do oscillator mode runs only in serial (single-processor) mode.

Nearly all oscillator input parameters are specified in the second namelist in_extra; setting losc $=. t$ or 1_extra=.t in the first forces the reading of the second namelist. Oscillator input parameters include: npass which specifies the total number of passes; npass_out (default value $\equiv 1$ ), the interval (in passes) to write the output radiation field to the postprocessor file; $\mathbf{z} \mathrm{m} 1 \mathrm{~m} 2$, the intra-mirror cavity length in meters; $r r_{\lrcorner} 1$ and $r c . m 2$, the radii of curvature in meters for the two mirrors respectively; rad_mr, the actual radius of the mirrors in meters; and $r_{-} c_{c}$ ity total surface reflectance (in power) of the two mirrors taken together. This value of $r_{\text {_cavity }}$ does not include the effects of the finite mirror size; hence, the net reflectivity will be less. The input parameter d_synch sets the cavity detuning length measured as a fraction of the slippage length $L_{s}$. Note that, following Colson's formalism, a positive value of d_synch shortens the actual cavity length so that radiation field energy near the head keeps arriving earlier in time relative to the head of the e-beam pulse in successive passes.

\subsection{Grid and Numerical Integrator Input Parameters}

\subsubsection{Simulation Grid}

For non-waveguide FEL simulations, GINGER uses a non-linear, expanding radial grid that near the axis is nearly linear in $r$ and then exponentially expands for large $r$. The outer grid boundary is given by rmaxsim in centimeters while the number of radial grid zones is set by nnd $\leq 63$. The region over which the grid is linear is controlled by the input parameter rlinear such that

$$
r_{n}=r l \text { inear } \times \sinh [(n-1) \zeta]
$$

where $\zeta$ is determined by the condition $r_{n n d}=$ rmaxsim. A good choice for rlinear is the expected radiation mode size. In waveguide mode, the gridding is uniform in the $\hat{y}$ plane. 


\subsubsection{Numerical Integrator Input Variables}

The most important numerical integrator input parameter is eps, the maximum normalized error allowed by the predictor-corrector for a step in $z$ to be successful. Typical values lie in the range $10^{-5}$ to $10^{-4}$ with the average step size being proportional to eps ${ }^{+1 / 2}$. One may also set the initial step size (dt) the lower bound on step size ( $\mathrm{hlb}$ ), and the upper bound on step size (hub, default value $0.5 \lambda_{w} / Z_{r}$ ), all measured in Rayleigh lengths. hub $m$ is the equivalent upper bound in meters, The final value $\mathrm{hlb}$ also has a lower bound set by machine accuracy. The input parameter maxstep is an upper limit to the number of integrator steps permitted during a full integration interval in $z$ ( $\equiv L_{w} /$ ncurve for FRED mode and $L_{w} /$ nsidep for polychromatic mode). If this limit is exceeded the code will abort. The default value is 10,000 and a user should normally not need to set maxstep any higher but there can be situations where one might need a larger value. Running with a very high value is potentially dangerous in terms of excessive CPU costs if the code begins acting bizarrely.

\subsection{FRED-mode Parameter Scanning Capability}

\subsubsection{General Parameter Scanning Input Variables}

On certain occasions, a user might want to know how sensitive a FRED-mode output parameter (e.g. power, saturation position in $z$, etc.) is to a particular input parameter (e.g. beam current, energy spread, etc.). One could follow a "brute force" approach and do a number of separate FREDmode runs with the individual input decks scanning the appropriate range in the desired variable input parameter. However, an alternative beckons due to the multidimensional nature of the timedependent GINGER pltfile, It was relatively easy to change the post-processor to produce a pltfile with the scanning variable (e.g. beam current) replacing time as the independent third dimension (with the first two dimensions being $r$ and $z$ ). Likewise, GINGER's monochromatic FRED-mode was then extended to be able to loop through the varying input parameter.

To use this capability, the input deck should be modified as follows: 1) nside should be set to the number of different values the input variable will take; in general this will probably be in the range 8-32 and need not be related to a power of two; 2) set lfred=.t to indicate a monochromatic FRED-mode scan rather than a polychromatic run; 3) set 1_extra=.t to force the reading of the \&in_extra namelist; 4) within the \&in_extra namelist, set the input variable param_name to one of the following allowed strings:

$\begin{array}{lllllr}\text { CURRENT } & \text { BRIGHT EMIT } & \text { EMIT_MKS } & \text { GAMMA } & \text { DGAM } \\ \text { AWO PLASER } & \text { R_MISMATCH } & \text { THETA_DRIFT } & \text { D_COLSON }\end{array}$

note: the variables may also be given in lower case (but not mixed case); 5) set the scanning range for the input variable by giving values to param min and param max in the \&in_extra 
namelist.

The default is a linear scan over the full range of the input variable. If $1 \_l o g$ param $=. t$, then the scan is done with a geometric scaling (i.e. equal increments in the logarithm as one probably would want to use if scanning PLASER over the range $1 \mathrm{~kW}$ to $1 \mathrm{GW}$ ). DGAM refers to scanning in the instantaneous energy spread while AWO is for scanning $a_{w}$ in a fixed (untapered) wiggler. Scans in $a_{w}$ can be very useful if the FEL parameter $\rho$ is quite small and one is trying to maximize gain in a situation where $2 \mathrm{D}$ or emittance effects are important.

\subsubsection{FRED-Mode Parameter Scanning with Multiple Processors at NERSC}

When running on one of the NERSC Cray-J90's (which have SMP architectures) or the Cray-T3E or IBM-SP (which have MPP architectures), it is possible to use multiple processors simultaneously for a significant speedup when doing a FRED-mode parameter scan. For the T3E or IBM-SP, one need only specify the wanted number of processors on the GINGER execute line (see $\S 2.5 .1$ ), making sure that nside is evenly divisible by the number of processors. For a J90, one must first set the UNIX shell environment variable NCPUS to a value greater than one; for example, setting setenv NCPUS 4 allows up to four processors running simultaneously for any executable (not just GINGER). It is not necessary that nside be evenly divisible by NCPUS. In interactive mode on a NERSC J90, one is not likely to get simultaneously more than 8 CPU's; in batch mode it may be possible to get more than $50 \%$ of the physically available CPU's if usage is light.

Within the main GINGER input file namelist \&in, one should set the input parameter ncpu to a value greater than one (this applies only to the Cray J90; ncpu is ignored for the T3E and IBM-SP). At present, if ncpu $>1$, it is best to set ncpu to a value $\geq$ NCPUS to prevent the possibility of idle processors (and a possible run abort) in the parallel control section of the code. The code will internally reset ncpu to be no greater than the value of NCPUS. If all goes well and if machine usage is light, one should get a speed-up in wall clock execution time of nearly a factor of $\min$ (NCPUS, ncpu). Since the FRED-mode scanning main loop is "embarrassingly" parallel, this multiprocessing feature does not involve the complexity of message processing from one processor to another. Also, in contrast to MPP machine multiprocessor mode behavior, one and only one output pltfile is made by a $\mathrm{J} 90$ in multiprocessor mode and there is no need for special concatenation following run completion.

\subsection{Output Diagnostics Control Variables}

There are relatively few input variables to GINGER which control the contents and details of the output files generated for later analysis by the post-processor code XPLOTGIN. When GINGER is run in a polychromatic, multi-slice mode for a single pass amplifier, radially-resolved radiation field quantities and various slice-averaged electron beam quantities are written at the end of every 
"interaction" interval in $z$, including $z=0$. Thus, there are nsidep +1 positions in $z$, nphoton positions in time, and nnd positions in $r$ for which both the real and imaginary components of $E$ are written to the pltfile. As explained in $\$ 3.4 .4$, one can set a switch in polychromatic mode to write out a special radiation field file for restart purposes. For all modes, macroparticle diagnostics written to the pltfile for each slice as a function of $(t, z)$ currently include the values of average macroparticle energy, the RMS energy spread, and the average bunching at the fundamental and possibly additional harmonics.

In single-slice FRED mode, dumps (i.e. snapshots at a given $z$ ) of the macroparticle phase space can also be requested (see \$3.11.2). In this mode, one can also request the creation of a simple ASCII datfile via the 1 datfile=.t which will contain simple columnar outputs of items such as the radiation power, macroparticle bunching, RMS delta gamma, etc. . For platforms such as DOS/Windows and LinuxX86 for which presently the post-processor has no direct graphical capability, this switch is default to .true.; however, it can be used for all platforms in single slice, FRED-mode. This file can be used directly by gnuplot as the non-data rows are begun by “\#\#”.

\subsubsection{Macroparticle Bunching Diagnostics}

Historically, GINGER has always computed macroparticle microbunching at the fundamental wavelength relative to the ponderomotive phase $\psi \equiv \theta+\phi$. The original rationale for this choice was that in many cases there could be significant radiation phase front curvature $\phi(r)$; this is especially true when the radiation mode size is significantly smaller than electron beam size. However, there may be reason to diagnose microbunching relative to a hypothetical plane wave (i.e. $\theta$ rather than $\psi$ ) and it is necessary to do so at higher harmonics (for which the field phase $\phi$ is unknown). Consequently, beginning in April 2001, the microbunching at the fundamental wavelength will now be calculated relative to a plane wave. To override this change and return to fundamental microbunching calculated relative to the ponderomotive phase, set the input variable 1_psi_bunch=.t.

Also beginning in GINGER versions of April 2001 and later, one can also output diagnostics of bunching at harmonics greater than 3 . The vector integer variable nhar_io should be set to the values wanted; note nhar_io $(1)=1$ (i.e. the fundamental) is hard-wired and cannot be over-

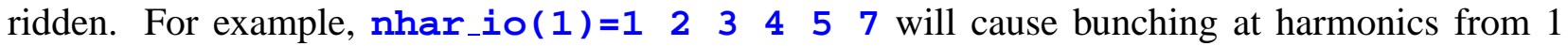
through 5 plus the 7th to be output. The bunching is a "complex" quantity with both amplitude and phase; a new feature of the postprocessor allows one to calculate the power spectrum $b(\lambda)$ of the microbunching (see $\S 4.5$ ).

\subsubsection{Macroparticle Phase Space Snapshot Output}

When GINGER is run in monochromatic "FRED" mode (lfred=.t), one uses ncurve to set the number of output positions in $z$. If phase space scatter plots are wanted (presently available 
for FRED mode only), a positive integer value of nspec causes GINGER to write out a spcfile containing such information at nspec $z$ locations which include both $z=0$ and $z=$ zmxmeter. As of fall 2001, the output longitudinal phase of the individual macroparticles is now measured relative to a hypothetical plane wave (i.e. $\theta_{n}$, where $n$ is the macroparticle index) rather than their position relative to the instantaneous ponderomotive well (i.e. $\psi_{n}$ ). To change this and get $\psi_{n}$ rather than $\theta_{n}$, set 1_write_psi=.t. One must also set the switch 1 -psi_bunch=.t as described in the previous paragraph.

\subsubsection{Controlling the $z$-Frequency of Output to the Diagnostic Pltfile}

For a polychromatic, non-oscillator mode run, GINGER by default writes the radiation field and macroparticle diagnostics for each slice at the end of every interaction (slippage advance) interval in $z$. One can reduce the $z$-frequency of these diagnostics (and thus the total size of the pltfile), by setting $\mathrm{n}_{-}$diag_mod to a value greater than one. A choice of three, for example, would produce output at the end of every third interaction interval for each slice. Runs with long optical klystron dispersive drift sections will generally produce output at fewer than (nsidep +1$) z$-locations (see §3.7.2). The postprocessor automatically deals with this change.

In multi-pass, oscillator mode, GINGER writes out field and particle quantities at only one $z$ location (the physical end of the wiggler) for each pass. This is true for both monochromatic and polychromatic oscillator runs. Hence, there is no $z$-resolved information within the wiggler. The input parameter npass_out controls the frequency at which diagnostic information is written to the pltfile. The default of npass_out $=1$ is equivalent to output being written every single pass; a choice of npass_out $=2$ would lead to output every other pass, etc. .

\subsection{Sample GINGER and XWIGERR Input Files}

The following input files were chosen as examples of the various types of FEL configurations which can be modeled with GINGER. They and some post-processor output CGM files are available with in the "tar" archive GNX_TEST . tar which is stored in the public HPSS space at NERSC described in $\S 2.2$.

\subsubsection{Monochromatic, "Paladin" Tapered Wiggler Self-Design: inpalSD}

This input file adopts "nominal" LLNL Paladin parameters and calculates the time-independent (i.e. monochromatic) power gain from a 10-MW input laser at $\lambda_{s}=10.6 \mu \mathrm{m}$ over a 80 -mm period,

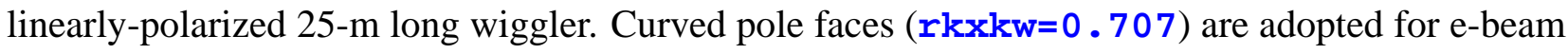
focusing. This run also uses the GINGER tapered wiggler self-design algorithm (via idesign=2) 
to compute $a_{w}(z)$ and sets the design macroparticle at $0.285 \mathrm{~cm}$. There will be 1024 macroparticles with only 2-fold longitudinal symmetry as we are not concerned with third harmonic growth. In addition to the normal output post-processor file (named pltpalSD), which has data at 51 $z$-locations, there will also be output a wiggler file (bwpalSD) containing $a_{w}(z)$, a macroparticle dump file (spcpalSD) containing 6-D phase space snapshots at six positions in $z$ down the wiggler (i.e. every 5 meters including at $z=0$ ). Note that the bunching and longitudinal phase space coordinates will be calculated using the ponderomotive phase $\psi \equiv \theta+\phi$ rather than the default of $\theta$. This problem requires 3.9 seconds on the Killeen Cray-J90 (SV1 processor), 13.8 seconds on the Cray-T3E, and 4.8 seconds on the IBM-SP machines at NERSC. To run this problem one would type: $\quad$ xginger $r=p a l S D$

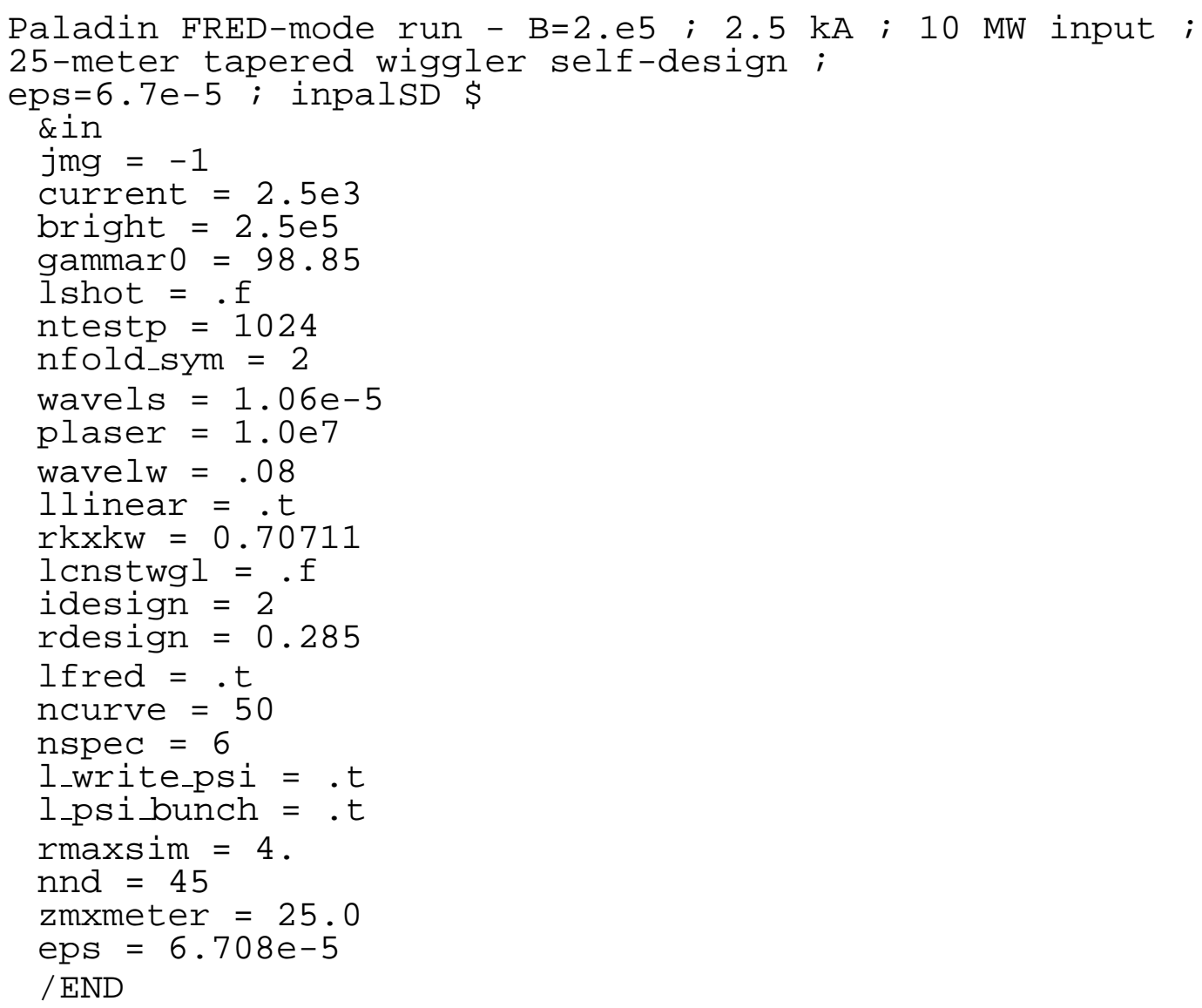

\subsubsection{Paladin Sideband Growth in a Tapered Wiggler: inpalacSTD}

This simulation initializes a white noise spectrum to provide a seed for sideband growth to a Paladinlike e-beam. With ampside $=0.01$ and plaser $=10 \mathrm{MW}$, the total noise power is $1.0 \mathrm{~kW}$. The 
input value $i d e s i g n=0$ and $\mathbf{l c n s t w g l = .} \mathbf{f}$ indicate that the wiggler is pre-determined, tapered, and will be read from an external file (bwpalac). By default, there are periodic temporal boundary conditions with 80 slippage advances over $25 \mathrm{~m}$ (or approximately every $30 \mathrm{~cm}$, much smaller than the gain length) and 96 separate e-beam slices. This example took 1250 seconds in serial mode (1 $\mathrm{CPU}$ ) on the IBM-SP, 230 seconds in 6-CPU parallel mode on the IBM-SP, 1280 seconds in 4-CPU parallel mode on the Cray-T3E, and 6630 seconds on a 400-MHz Pentium II under Linux86. To run this case on a single processor, the user would type: xginger r=palacSTD b=bwpalac To run this case on the Cray-T3E employing 4 processors in parallel, the execute line would be:

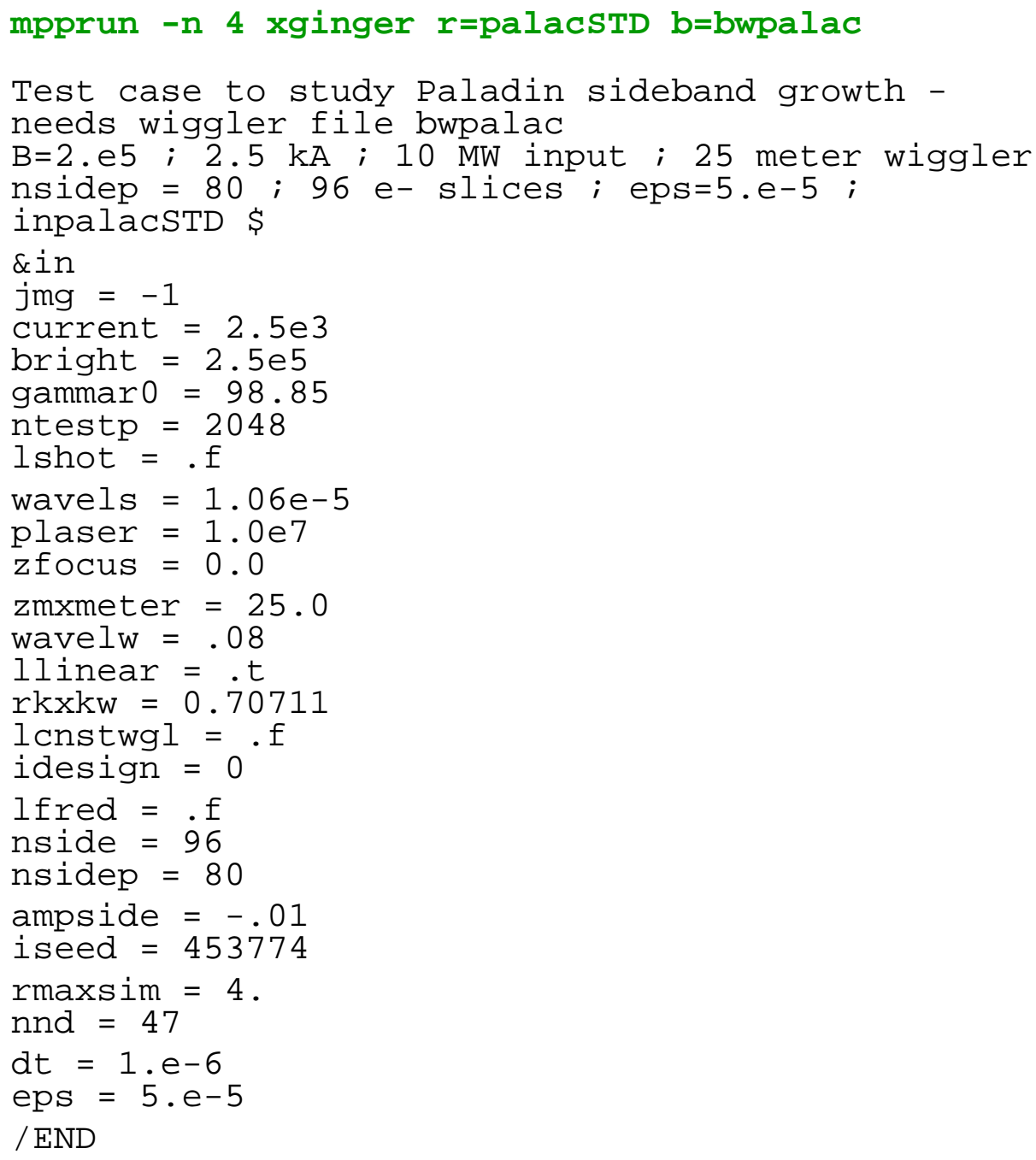




\subsubsection{Long pulse, LCLS 1.5Å SASE x-ray FEL: inlcls_fodoSb}

This particular input file sets up a full polychromatic simulation of the proposed LCLS x-ray FEL at SSRL/SLAC, operating at 1.5 Angstroms with a $15-\mathrm{GeV}$ electron beam. The temporal boundary conditions are periodic by default. Each electron beam slice has a small amount of instantaneous energy spread. Although particle shot noise provides the dominant seed for radiation growth, a small level of white noise power is put in at $z=0$ via the wattpbin input parameter; this is needed at present to keep the predictor-corrector happy at $z=0$. Note that the random number seeds for both the shot noise and the random radiation field noise are preset by the user. The longitudinal window length is set by nsidep, the total number of slippage advances occuring for each of the 192 electron beam slices over the full wiggler length. The linearly polarized wiggler has a constant $a_{w}$ strength whose on-axis value is preset by the input variable aw0. The initial transverse phase space orientation for the electron beam in each plane is set by the input values of the Twiss parameters. The external focusing is a FODO-type lattice with a period of 4.31 meters. Each magnet is associated with a drift space whose length is 0.135 meters. The radial simulation grid is approximately linear out to 40 microns and has an outer boundary of 150 microns. The full simulation length (including drift spaces) is 112.5 meters with an initial stepsize of $10^{-6}$ Rayleigh ranges, a minimum stepsize of $10^{-8}$ Rayleigh ranges, and an upper step-size limit of 0.03 meters (this ensures each quad magnet will be "hit" at least once by the adaptive $z$-stepsize integrator). To run this case (on a single processor), one would type: xginger $r=l c l s \_f o d o S b \quad$ To use 8 processors on the IBM-SP (presuming the MPP version of the GINGER executable is named xginger-mpp), one would type: $\quad$ xginger-mpp -procs 8 r=lcls_fodosb

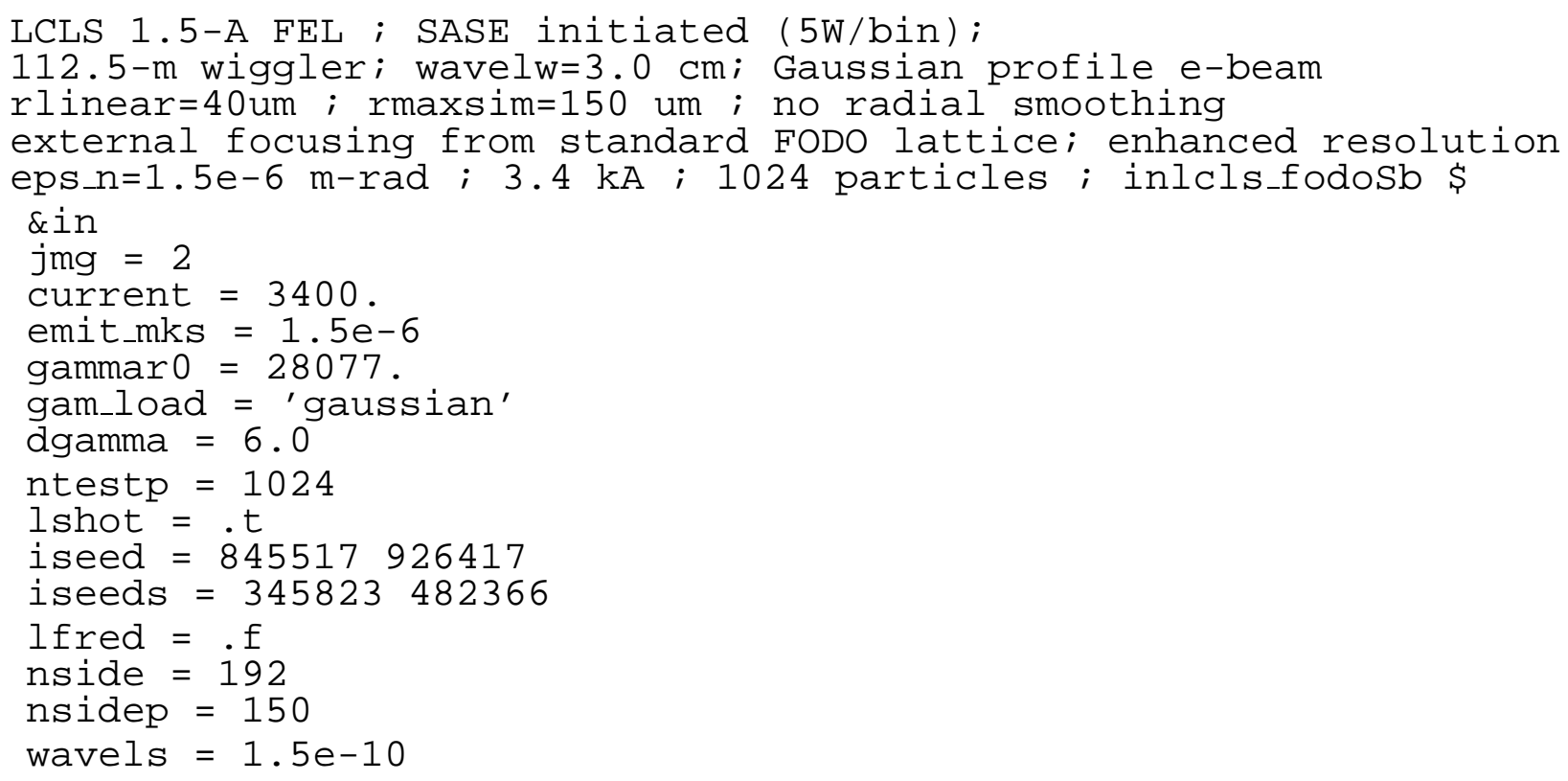




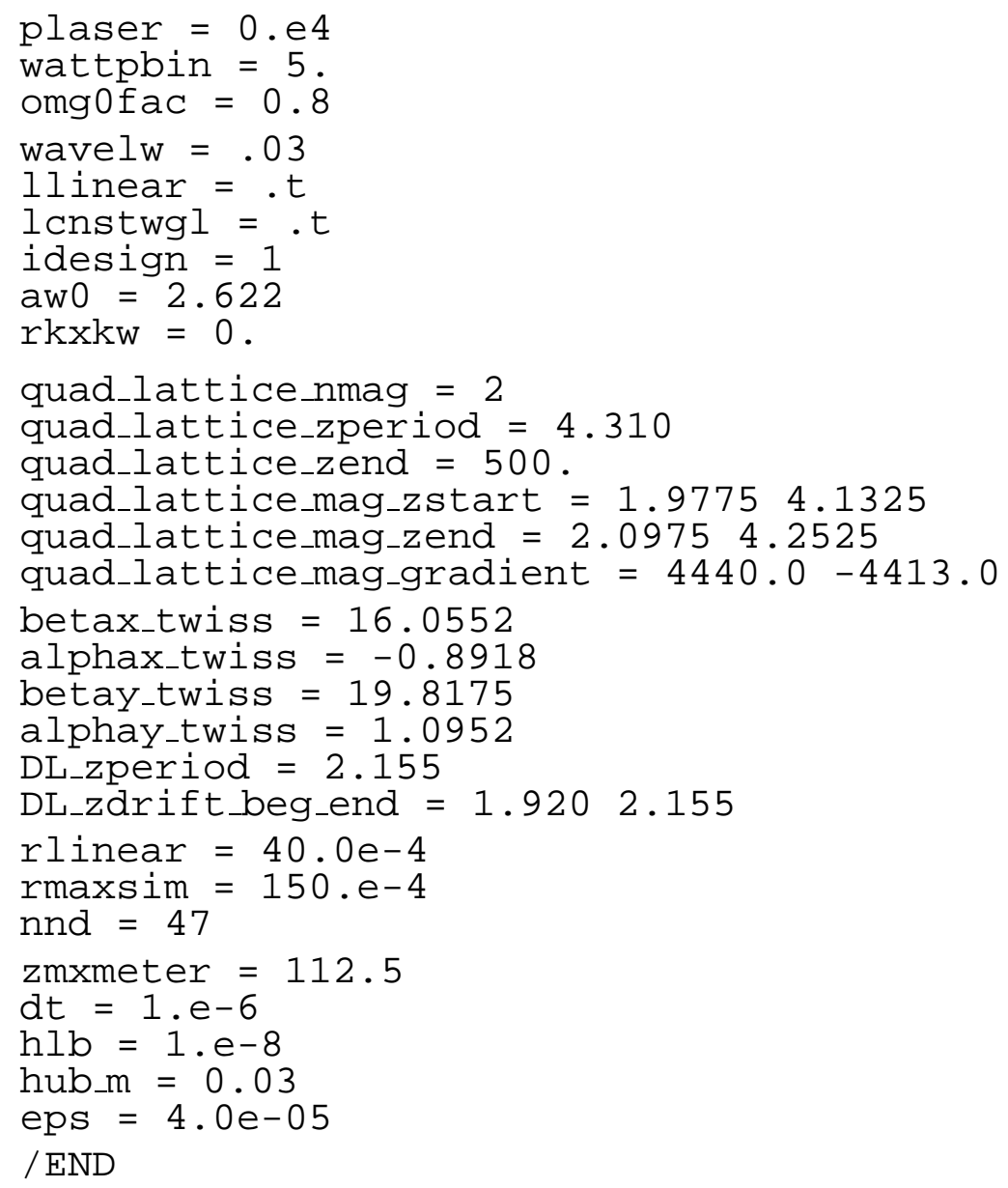

\subsubsection{Sample LCLS Wiggler Error Input File for XWIGERR Program}

This input file is used by the XWIGERR program to generate wiggler and quadrupole errors and corresponding steering for an LCLS run with parameters similar to the previous input file. The output file lattice_file lcls_bpm-50off.lat can subsequently be used by GINGER. The net effects of the pole errors are averaged over 6 wiggle periods, which leads to an output interval of 18 $\mathrm{cm}$. Note that one needs to specify mag_units $=1 \mathrm{G} / \mathrm{cm}^{\prime}$ for the input quad magnet gradients because the default for XWIGERR is T/m. The steering lattice has a $4.31-\mathrm{m}$ period which begins at $2.04 \mathrm{~m}$; there is an additional steerer at $z=0$. The rms offset error for the beam position monitor is set to 50 microns.

sample input file for XWIGERR program

LCLS-type undulator; 0.16-period undulator period averaging 


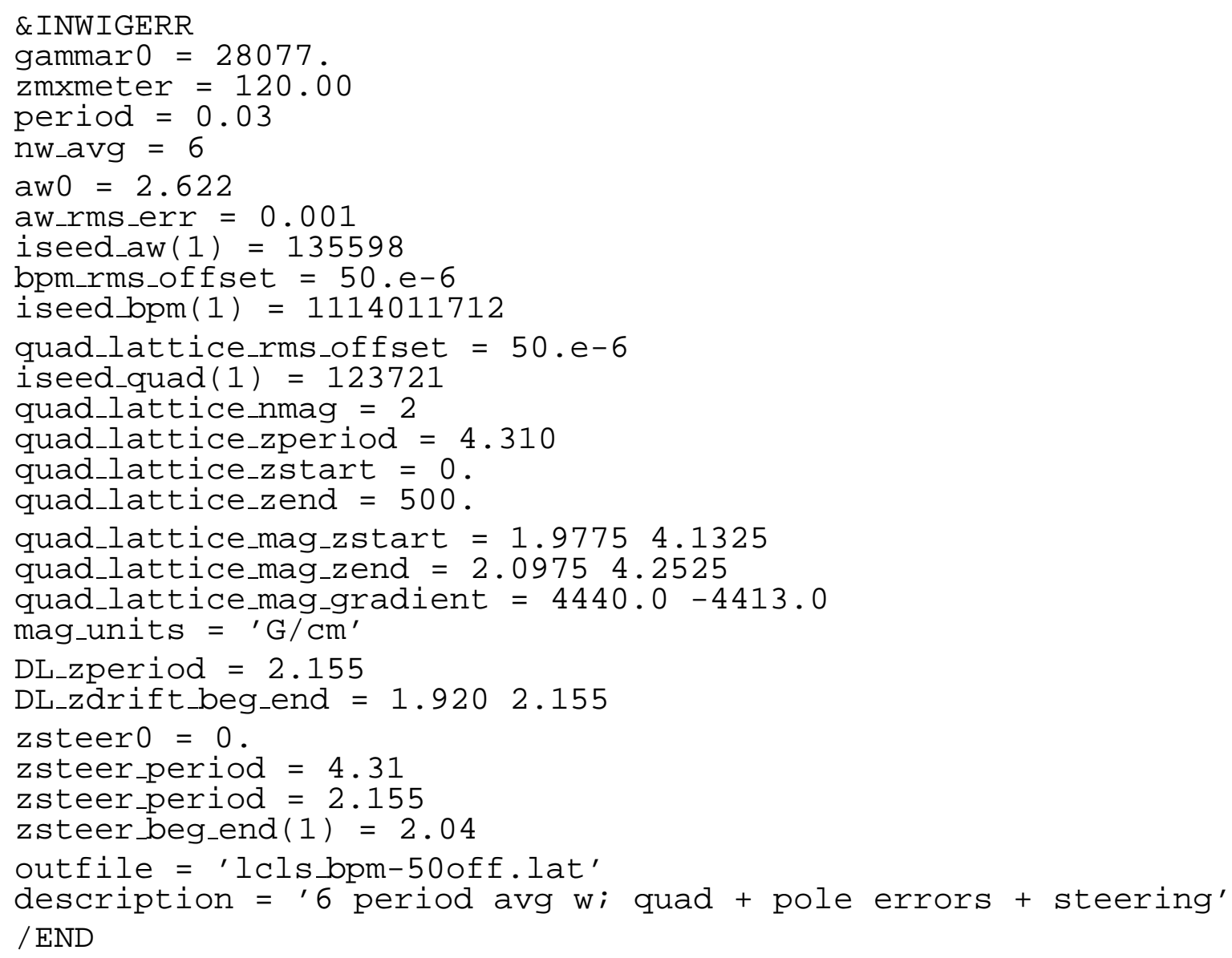

\subsubsection{Example LCLS-case Template-File Use: inlcls-errA2}

This input file relies upon the use of inlcls_fodosb as a "template" file and extends it by using the wiggler error file produced in the previous example. This file (via setting dgamma) also lowers the energy spread to 3.0 from the template's 6.0, the normalized emittance to $1.0 \mathrm{~mm}$-mrad, increases the number of macroparticle's to 2048, resets the random number seeds for the energy spread and shot noise, and adds an $x$-offset of 30 microns to the initial ebeam centroid (remember, GINGER nominally uses centimeter units for transverse quantities such as xoff and yoff). To run this example on a single processor, the user would type: xginger $r=l c l s-e r r A 2$ t=inlcls_fodosb

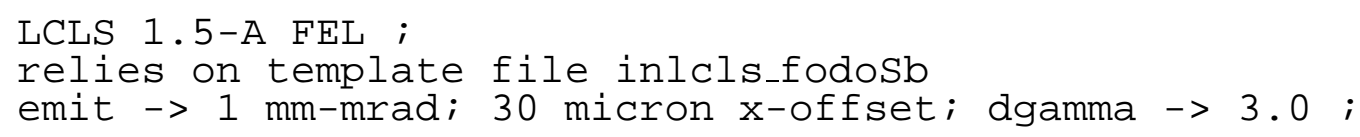




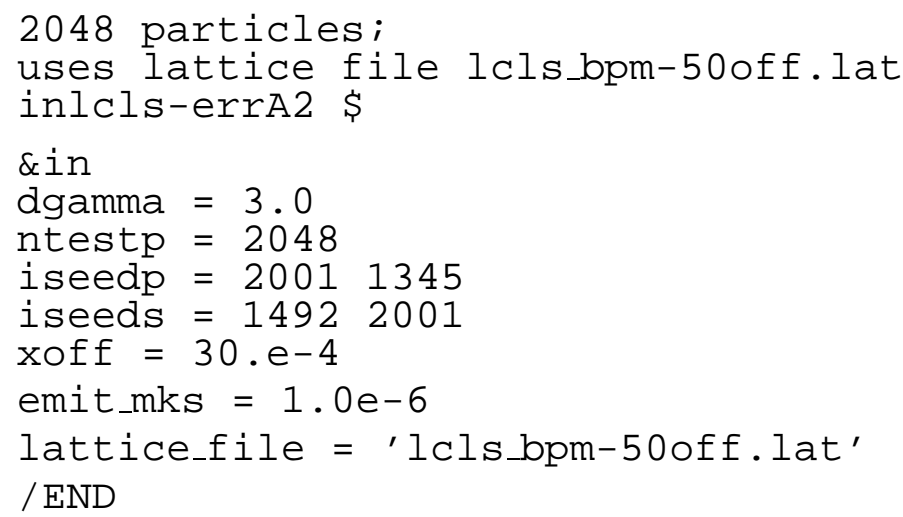

\subsubsection{Short pulse, UCLA single-pass SASE expt.: inUCLAt2}

This input file is used to simulate the behavior of the mid-1990's UCLA SASE IRFEL which operated at a central wavelength of 10.6 microns. This is a "short-pulse" simulation as set by ltransit=.t. With a period of $15 \mathrm{~mm}$ and $0.6-\mathrm{m}$ length, the wiggler is 40 periods long and, choosing nsidep $=20$, the photon slices advance forward one electron beam slice every two wiggle periods. With nside $=60$, the electron beam is 3 slippage distances long, which is equivalent to 1200 microns or 3 ps in time. The electron beam has a parabolic current profile (via pulse_shape) in time. nphoton=96 is greater than $n s i d e+n s i d e p+1$ as required. Shot noise is initialized via 1 shot =. $t$ and the intial radiation is a flat spectrum with 100 nanowatts per bin. There is no external focusing in the wiggler plane, but there is presumed to be a simple lens at $z=0$ whose focal length in the wiggle plane $(\hat{x})$ is set to 0.35 meters via xfocus_mtr. The initial beam size in $x$ is $0.03 \mathrm{~cm}$ (=300 microns); the $\mathrm{y}$-size is set to be in equilibrium with natural wiggler focusing. This is a relatively fast running problem and took a total $850 \mathrm{CPU}$-seconds on the NERSC IBM-SP in parallel mode. The execute line to run this problem is: xginger r=UCLAt2

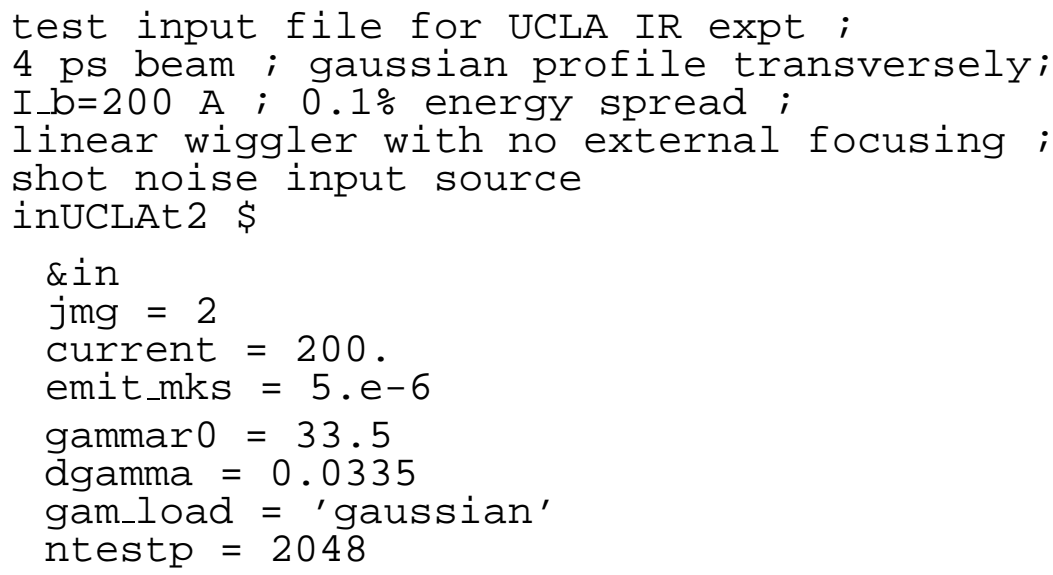




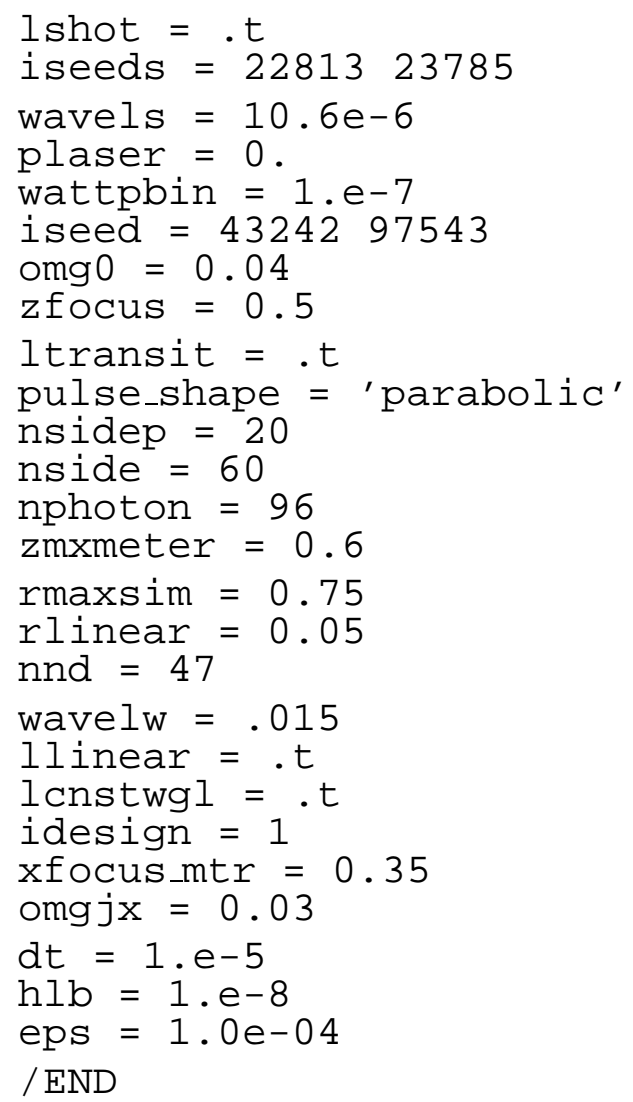

\subsection{Names and Default Values for GINGER Input Namelist Parameters}

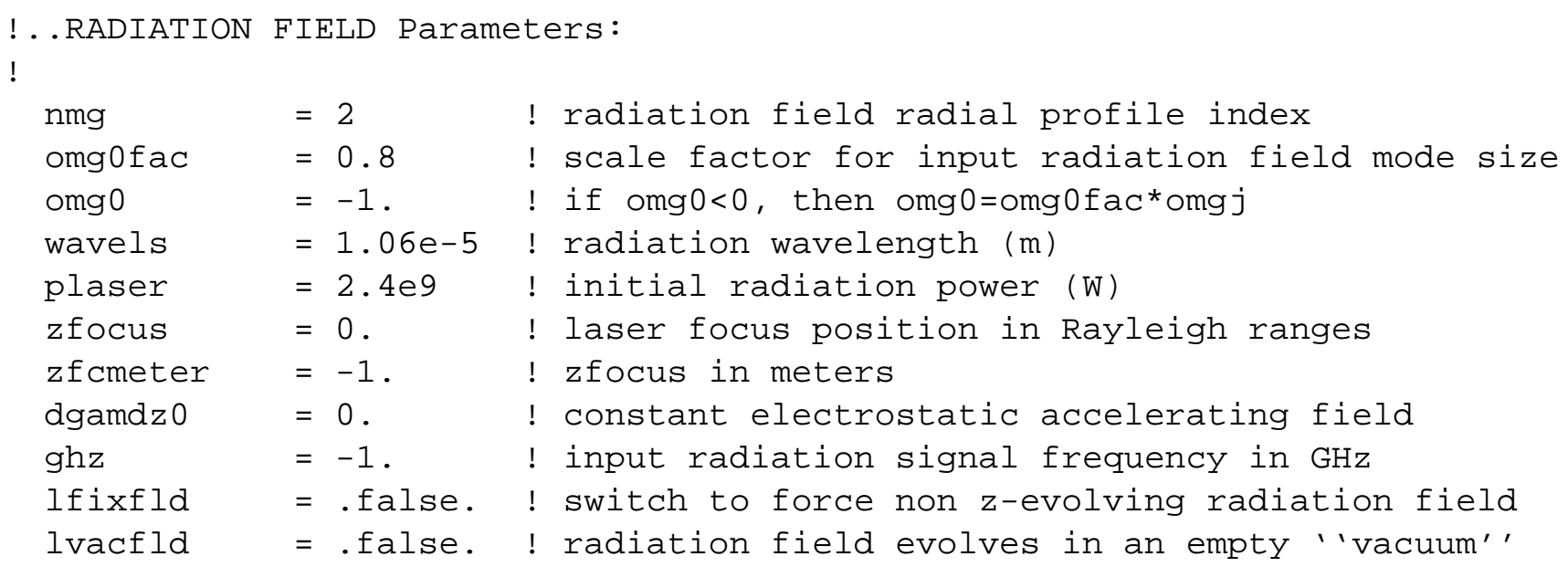




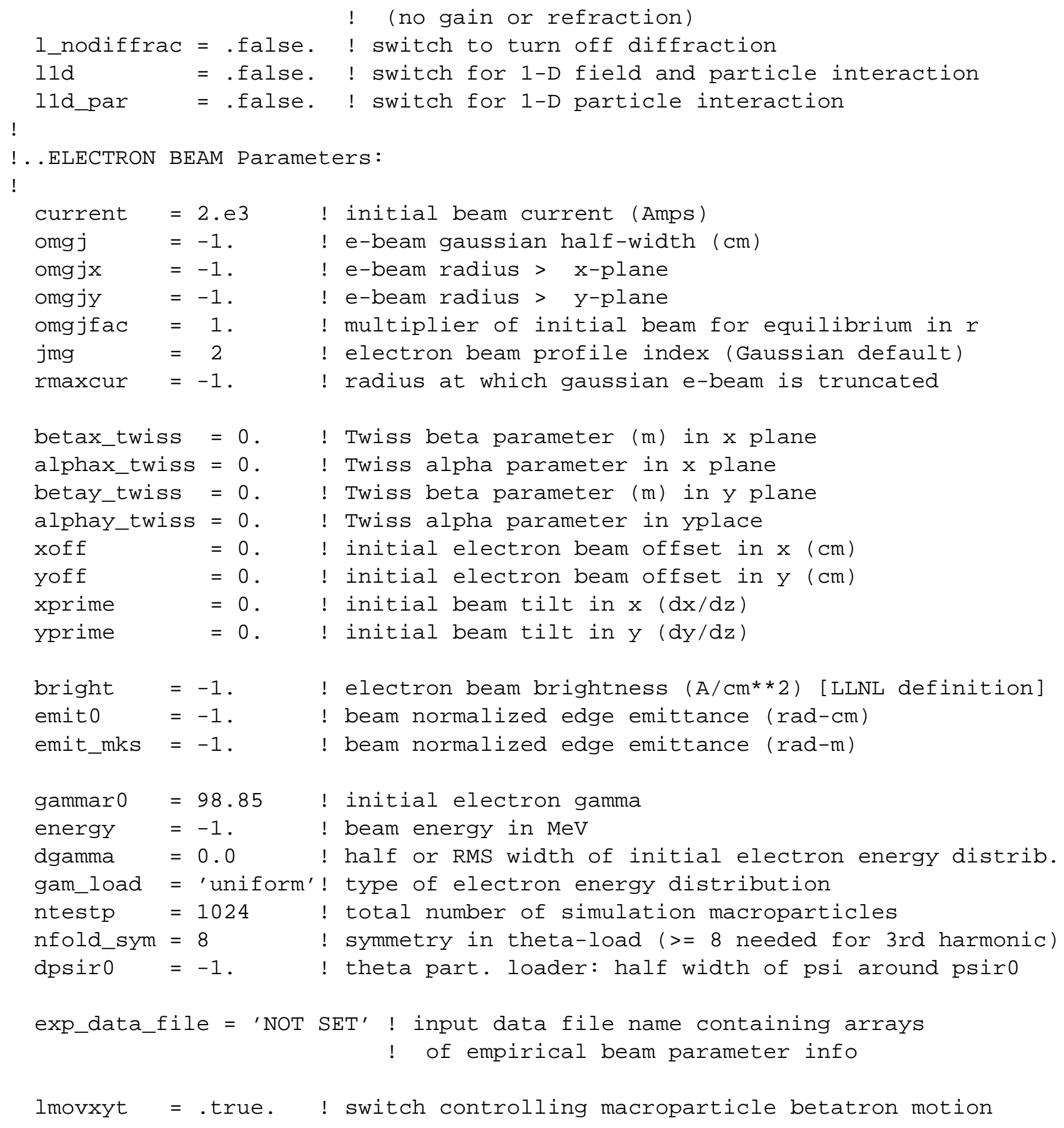




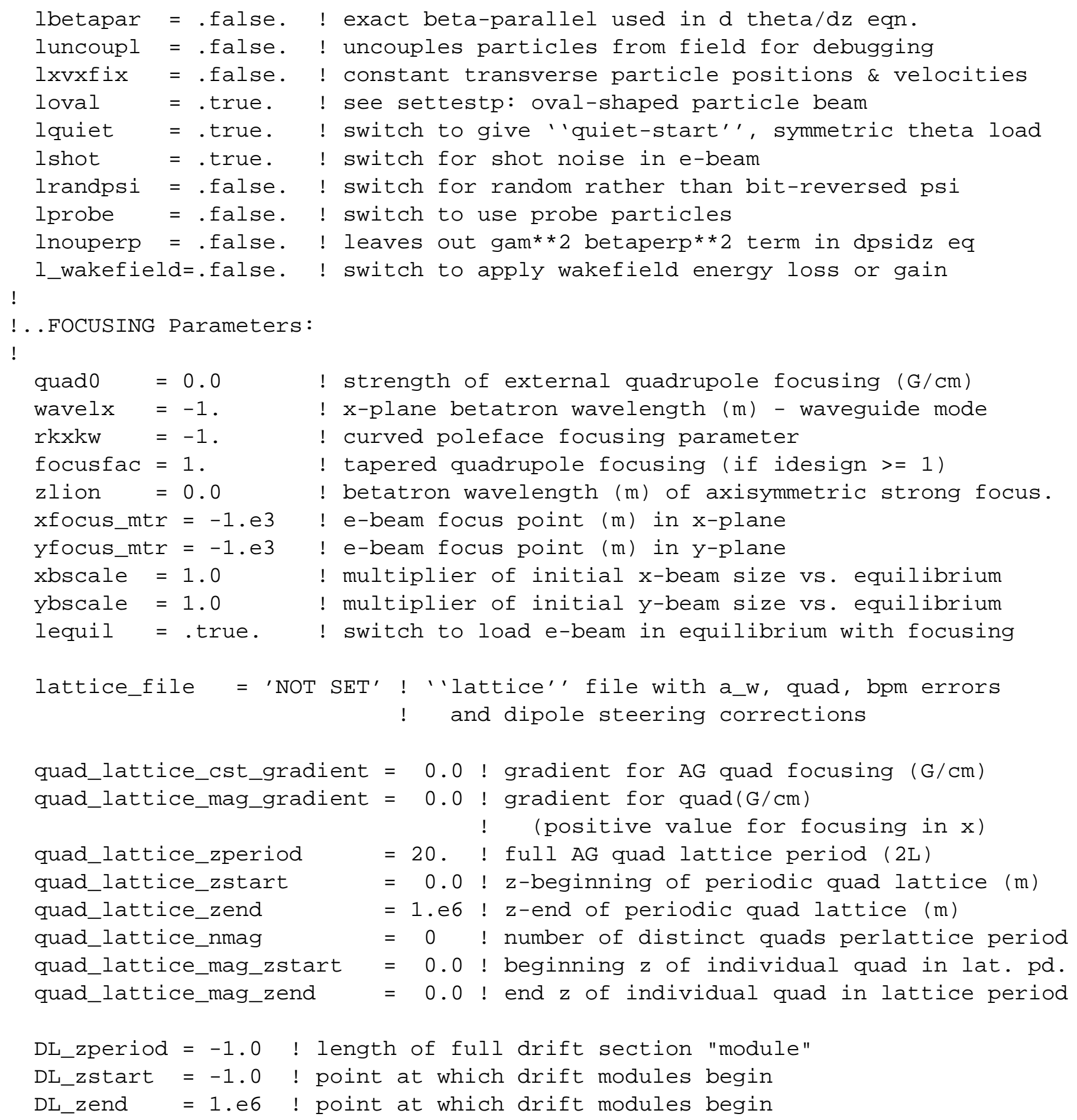




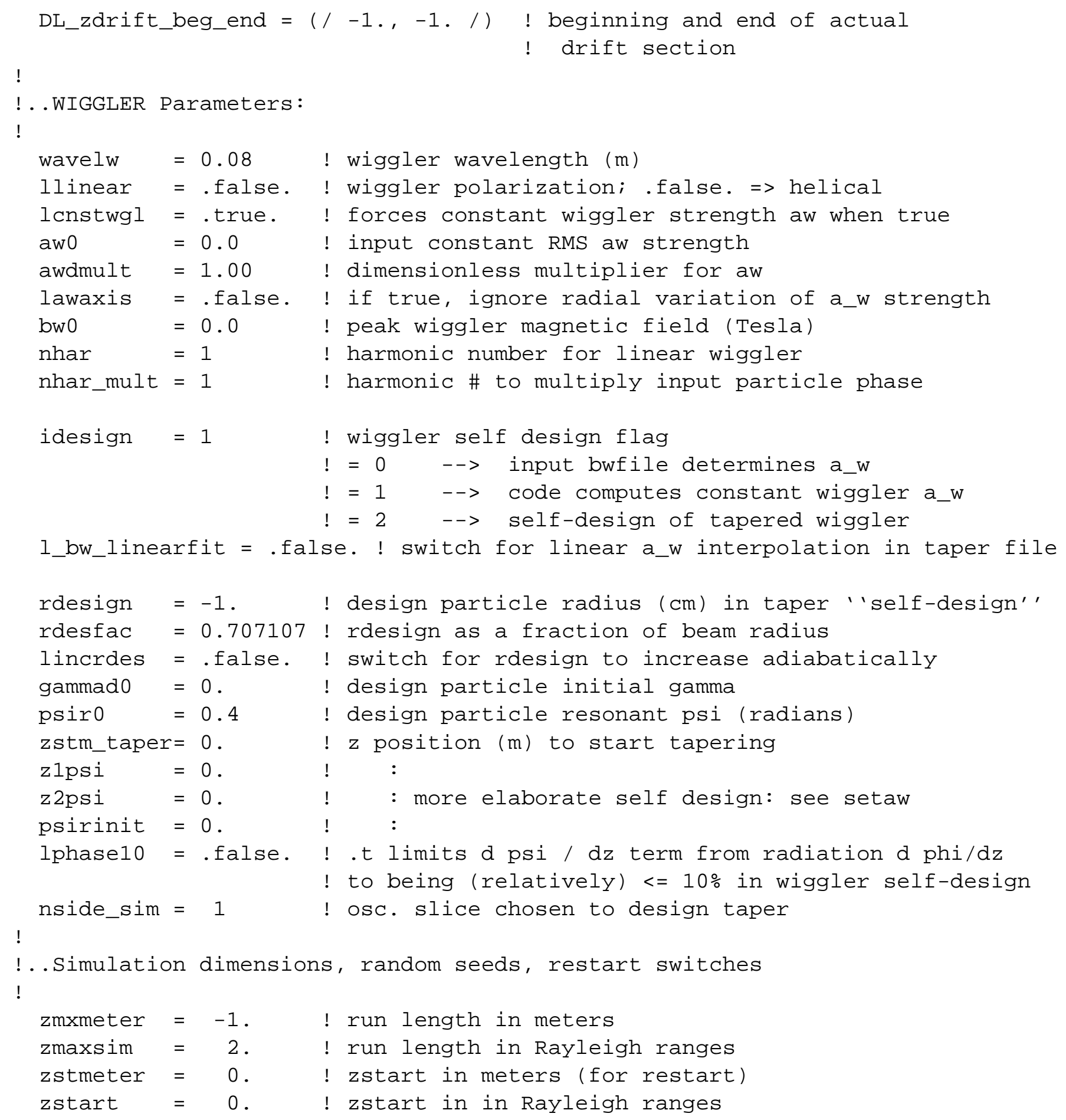




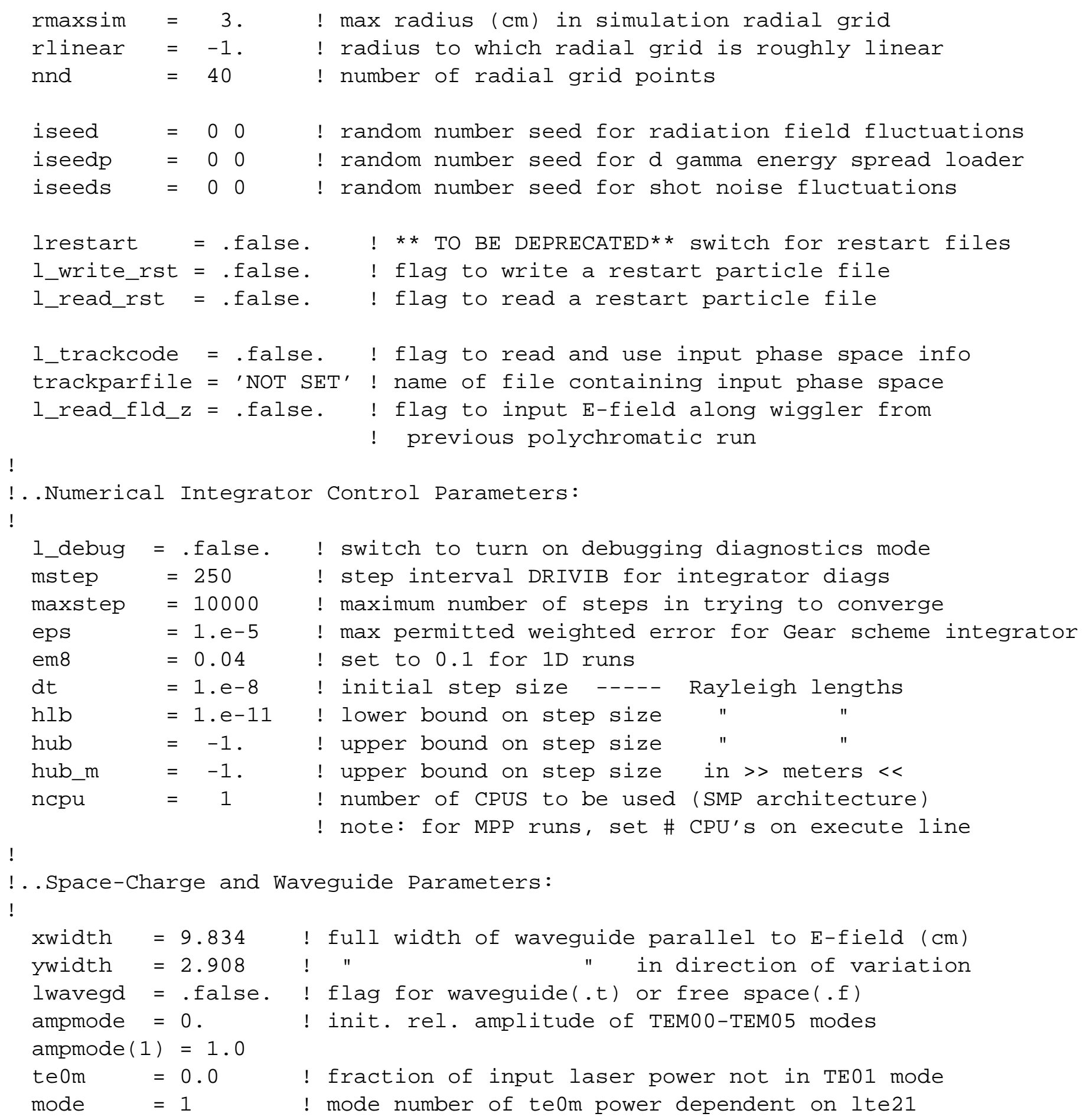




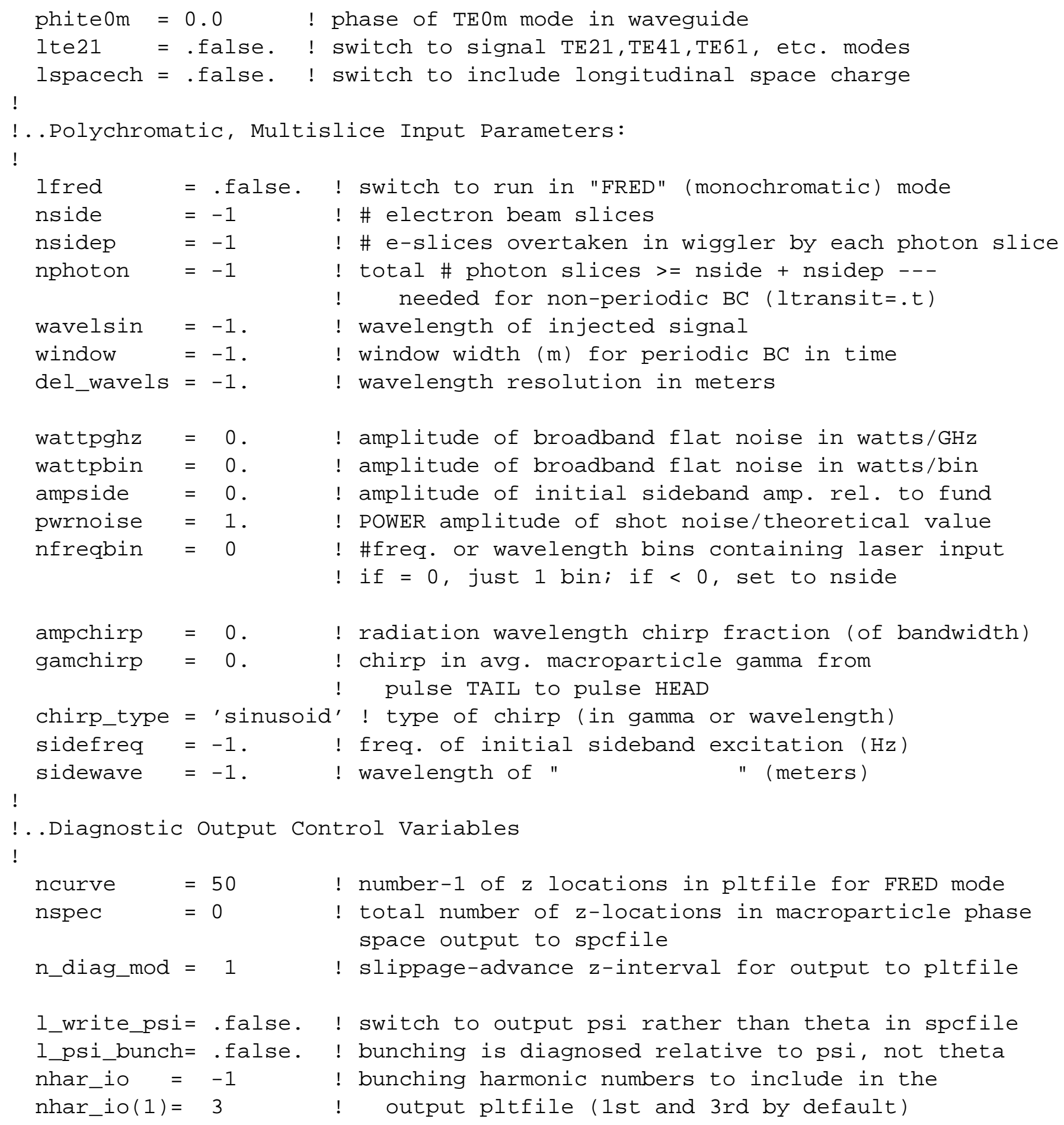


l_write_fld_z= .false.! flag to write z-dependent E-field of head e- slice

! for purposes of a later pulse-extending restart

! or single slice FRED-mode run

l_datfile =.false. ! flag to write simple ASCII output file with

! variables such as radiation $P$, bunching, etc.

!

!.. Short Pulse Mode (non-periodic BC) Input Parameters:

!

Itransit $=$.false. ! switch to put in non-periodic $B C$ on rad. field

pulse_shape $=$ 'tophat'! longitudinal e-beam pulse shape

trise $\quad=-1 . \quad$ ! current rise and fall time in sec

tbody $\quad=-1 . \quad ! \mathrm{FW} 1 / \mathrm{e} \max$ of current in sec or RMS pulse

! duration for gaussian profile

!

!..Oscillator and Optical Klystron Parameters:

!

l_extra = ffalse. ! switch to read "inextra" input namelist

losc $=$ false. ! switch to tell code if an oscillator run

npass $=1$ ! \#asses in oscillator

npass_out = 1 ! interval in pass \# for writing output diags

r_cavity = $1 . \quad$ ! reflection coefficient for loss in cavity

d_synch $=0 . \quad$ ! relative (to slippage) cavity detuning length

z_m1_m2 = 12.5 ! cavity length in meters

rad_mr $\quad=0.01$ ! radius of mirrors in meters

rc_m1 = 7.5 ! radius of curvature of first mirror in meters

rc_m2 = 7.5 ! radius of curvature of second mirror in meters

fldfac $\quad=-1$ ! factor to multiply input radiation field power

del_jlstrt $=0$ ! offset to move photons in restart

nuo_colson $=0 . \quad$ ! norm. offset of particle energy a la Colson

d_colson $=0 . \quad$ ! OK drift dispersion parameter

OptKly_zlen $=0.5$ ! physical length $(\mathrm{m})$ of OK drift for opt. propag.

OptKly_zwgl_equiv $=-1 . !$ OK length in terms of equivalent wiggler

t_OKdrift $=-1 . \quad$ ! dispersive length in Rayleigh ranges

z_OptKly $=-1 . \quad$ ! location (m) of drift section for OK 


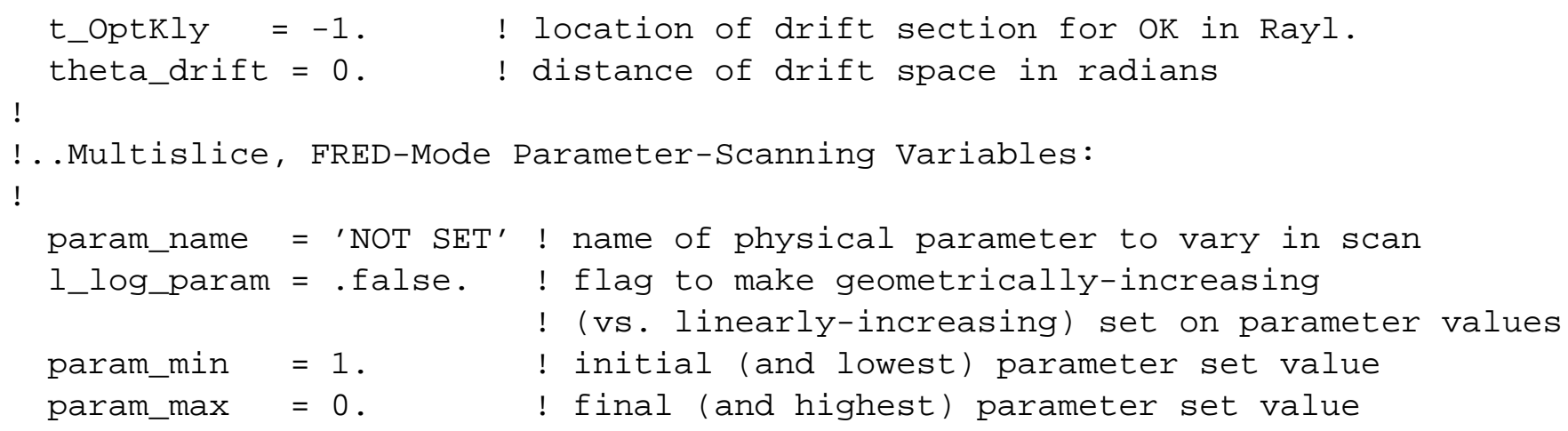




\section{4 “Preferences" File for the XPLOTGIN Post-Processor}

\subsection{General Information}

The postprocessor XPLOTGIN employs an optional ASCII "preferences" file to set various plotting options, including producing output in forms (i.e. ASCII, SDDS, HDF datafiles) other than graphical. The default name for the preferences file is xplotgin.pref. Beginning in fall 2001, however, alternate files may be specified by typing the XPLOTGIN execute line option pref=' 'mypfile' ' where ' 'mypfile' ' represents the wanted preferences file to be read by XPLOTGIN. The preferences file must exist (in actuality, or via a hard or "soft" UNIX link) in the same directory from which XPLOTGIN is being run. The preference file should contain a Fortran90 namelist called inpref whose variables can control much of the behavior of XPLOTGIN. Some options have effects on all GINGER runs, others only for polychromatic runs, and a few apply only to monochromatic (FRED-mode) runs. Recent work on preference-file namelist options has concentrated extending the output data file generation capability to allow users to use other visualization tools (e.g. gnuplot, sddsplot) to analyze and display GINGER simulation data, and to control various details of the diagnostic plots. This work is continually ongoing and the user should attempt to always use the most recent version of the postprocessor (which also avoids possible compatibility issues when using a very recent version of GINGER).

\subsection{Graphics Output Device, Color, "Logo" Control Variables}

The preference file variable lnoplot can prevent the creation of the NCAR graphics file (be it X11, CGM or Postscript output). One normally sets lnoplot=.true. only if, rather than graphical output, you only want output data (via the 1 datfile or 1 hdf switches for example) in ASCII or HDF files. You could also use this option to make sure a given pltfile is readable and analyzable by XPLOTGIN. Setting 1_color= . false creates monochrome (B\&W) graphical output; one might use this option when needing Postcript output for a scientific paper and/or for later conversion to PDF format. Similarly 1_logo=. false. prevents the "logo"text (which contains the GINGER run name and date) from being placed in the upper right-hand corner of the individual plot frames. To minimize the total number of plots generated, keep $\mathbf{l}_{-} \mathbf{f u l l p l o t s}=\mathbf{f} \mathbf{f}$, the normal default. This will skip various plots most users generally do not use, i.e. 3-D contour plots of $I(r, z), \phi(r, z)$, and obscure plots like the synchrotron wavenumber, e-beam power loss, etc. . Many of these plots can be turned on individually via other preference file input variables. 


\subsection{Pltfile Input Read Control Variables}

One seldom needs to use any of these variables as they were developed mainly to deal with "faulty" pltfiles, e.g. one generated by a GINGER run that did not finish to completion. For example, if an oscillator run did not reach the final pass specified by the value npass in the original GINGER input file (and which is echoed in the beginning of the pltfile), the preferences variable npass $i$ will override this value. Similarly, nsidep_i and nphoton $i$ overide the pltfile values of nsidep and nphoton, respectively. However, it remains somewhat hit-or-miss whether the latter two variables will allow the postprocessor to analyze a faulty pltfile properly. The variable $1 \_$read_3rd_har $=. \mathbf{f}$ instructs XPLOTGIN not to to read 3rd harmonic bunching data. This capability exists to ensure compatibility with quite old pltfiles which did not write such bunching data; most users will never use it.

\subsection{Radiation Power and Bunching Plot Control Variables}

Setting 1_plot_engbal=.t generates plots of total energy balance versus $z$. To force the ordinate scale on macroparticle bunching snapshots to be 1.0 rather than the local (in $z$ ) maximum, set 1 _bunch max =.t. Setting 1_plot_3rd_har $=$. $\mathbf{f}$ suppresses plots of 3rd (and higher if they exist) harmonic bunching fraction. To suppress plots of the instantaneous energy spread, set the switch 1_plot_delta_gamma=.f. To set the specific plot locations in $z$ for the bunching and radiation power snapshots versus $t$, use the real 1D array zsnap_plot; the "deprecated" variable z_snap previously controlled these locations. Presently, up to 9 values are allowed with default locations being evenly spaced through the simulation's range in $z$. One can change the variable ifirst_testp from its default value of 1 to specify the index of the first snapshot plot $z$ location; this is generally appropriate for shot-noise-initiated runs in which the initial radiation field is essentially zero (the preferences switch $1_{-}$sase $=. t$ also does this).

To generate radial profile plots of the radiation intensity, set 1_plot_radial_profile=.t. Currently, these plots will be at the same $z$-locations as the power spectrum plots (see the next section). By default, these intensity profile plots are accompanied by plots of the ("slowly-varying") radiation phase $\phi(r)$; the latter can be separately turned off by setting 1_plot_phase_r=. $\mathbf{f}$. To generate contour plots of the intensity, set 1_3Dplots=.t. Section 4.8 discusses some additional preference file variables which control generation of SDDS files with intensity and electric field information.

The switch 1_plot_farfield controls the calculation of and plotting of the far field radiation pattern. As of October 2001, this has a new default value of . false.. 


\subsection{Spectrum Plot Control Variables}

The switch 1_plot_spec, normally true, can be used to turn off all plots involving spectra. A number of variables can modify the number and $z$-locations of the power spectrum plots $P(\lambda)$. Normally there are 5 such plots evenly spaced in $z$; the preferences variable n_spec_plot can overide this. Alternatively, the real 1D array zspec_plot can override even spacing and set the actual $z$-locations. Up to 16 values may be input but remember there are only nsidep+1 available in the simulation. The equivalent array to control $z$ locations in ASCII/SDDS output files is zspec_print. The real array zgain_plot will set z-locations at which the "instantaneous" gain $(\mathrm{db} / \mathrm{m})$ is plotted versus wavelength. By default such gain is averaged over a $z$-interval of one-tenth the simulation length; this averaging length can be changed by giving a value in meters to the preferences variable $\mathbf{d z} \mathbf{z}_{-}$gain.

Normally, the power spectrum plots are produced in a semi-log format. This can be changed to a linear scale by setting 1 linear_specplot=.t. The variable spec_pwr_plot_limit can set the range in watts of the spectral plot ordinate. The default in semi-log format is to allow up to 8orders of magnitude in the range with the max and min values being exact powers of ten. To change the wavelength interval in the power spectrum plots from the default value of the full simulation bandpass, give the wanted spectral range in meters to the two-element array wspec_plot_limit. Setting the variable $\mathbf{n}_{\text {avg_spec }}>\mathbf{1}$ instructs the postprocessor to average the spectral intensity over multiple neighboring frequency bins for smoothing purposes.

Beginning in fall 2001, XPLOTGIN now has the capability to generate power spectrum plots of the calculated electron beam microbunching in the longitudinal direction. These spectra can be compared with experimental measurements of coherent transition radiation. They will be generated if either 1_plot_bunchspec=. $t$ (in which case they will be plotted at the same locations as the radiation spectra) or if the user gives $z$-location values for the real array zbunchspec_plot.

For time-dependent runs starting from noise, setting $\mathbf{l}_{-} \mathbf{s}$ s se $=. \mathbf{t}$ will start many plots versus $z$ at the second output $z$-location where true incoherent noise effects should dominate over the initial (coherent) radiation power (which the user presumably set to a very low value). This switch also suppresses plots of the upper and lower sideband growth versus $z$. One should not use this switch in a MOPA configuration where there is a strong input source. When $\mathbf{1}_{-}$plot_fund $=. \mathbf{f}$, the power at the fundamental (i.e. central) wavelength is "suppressed" in spectrum plots; this option is useful if one wants to examine sideband development in a single pass amplifier with a strong input signal strongly dominating the sideband power.

To generate plots of the temporal autocorrelation function for the radiation electric field at various $z$-locations, set 1_autocorr $=. t$. As of spring 2001, the on-axis, far field complex amplitude is used in the autocorrelation calculation. The number of such plots is controlled by nplt_cor with a default of value of 5; alternatively, the user may choose the $z$-locations via the 1D array zautocorr_plot. 


\subsection{Generating ASCII Output Tabular Data Files}

Several users have requested the ability to output simple ASCII files of variables such as $\langle P(z)>$ for input to other visualization and/or analysis codes. This capability has been significantly extended in XPLOTGIN over the last two years. It also is the only way presently to obtain post-processor output on platforms (e.g. DOS/WINDOWS) without access to NCAR graphics. To enable either simple tabular output (or SDDS-formatted file generation as described in $§ 4.7$ ), set the variable 1_datfile $=. t$ in the preferences file.

Non-SDDS output files are given names that begin with the GINGER run name followed by a mnemonic string indicating the physics type of data included therein. For example, if the GINGER run_name is palac, the files palac_data.zhist, palac_data.time, palac_data. spec will be output. At present depending upon the type of GINGER run, 3 types of simple ASCII tabular files can be generated:

' *_data.zhist' - this file contains separate columns of the output $z$-location, time-averaged radiation power, bunching at both the fundamental FEL wavelength and the third harmonic, rms radiation beam size, far field radiation mode size, and (in polychromatic mode) inverse rms spectral bandwidth.

' * data.time' - this file contains time-resolved data at the wiggler end and is thus only generated for GINGER runs done in polychromatic mode or monochromatic, parameter scanning FRED-mode, (see section 3.10) where the scanning variable (e.g. $a \_w$ ) replaces time as the independent variable. The first column contains the independent variable value, followed by separate columns for radiation power, electron beam power loss, and bunching at the fundamental FEL wavelength.

' *_data.spec' - this file contains wavelength-resolved radiation power spectra data at different individual $z$-locations and will be generated only for polychromatic GINGER runs. The locations can be set by the preferences variable array zspec_print, which may contain up to 8 values. If zspec_print is not input, there will be only one location, at the wiggler exit. The first column will contain the wavelength while the successive columns contain the power per wavelength bin at the different $z$ values.

\subsection{Generating SDDS Format Output Files}

The Self-Describing Data System (SDDS) is a data format developed by M. Borland and is presently in wide use at the APS facility at Argonne National Laboratory. An important and attractive feature of the SDDS package is the existence of numerous processing and plotting tools which can be used directly to analyze data written in SDDS format; see the URL 
http://www.aps.anl.gov/asd/oag/oaghome.shtml and associated links for documentation and download information concerning SDDS software. In late 2000, SDDS file generation capabilites were first implemented into XPLOTGIN and have been significantly extended in 2001. When both 1 _datfile=.t and 1_sdds_output $=. t$ in the preference file namelist, output data of the type described in section 4.6 will be written into SDDS files. The SDDS output files are in ASCII and contain information essentially identical to that stored in ASCII format. Each will have a suffix of .sdds (i.e. palac-time.sdds, palac-spec.sdds, palaczhist.sdds). If 1 sodds_output=.t, only SDDS format files (and not the ASCII-style files described in the previous section) will be output.

\subsection{Generating "Special Purpose" SDDS Output Files}

In addition to the "general" SDDS files mentioned in the previous section, one can also generate "special"-purpose SDDS output files. For these files, it is not necessary that 1 _sdds_output $=. t$. Setting the input variable pwr_lambda_print to an array of radiation wavelengths $\lambda_{i}$ (units in meters) will generate an SDDS file with the name run_name-pwr_lambda.sdds containing separate columns of $P\left(\lambda_{i}\right)$ versus $z$. If 1_write_inten_Rz =.t. an SDDS output file containing the time-averaged values of intensity $I(r, z)$ will be created. When zefld_print is set equal to an array $z_{i}$ of $z$ locations, the slowly-varying, complex, time-dependent electric field $\tilde{E}(r, t)$ (see $\S 5.1$ ) will be written out as a series of arrays to a file named run_name-efld.sdds. A final option l_write_fld_z controls generation of single slice radiation fields and is described in $\S 4.11$.

\subsection{Generating HDF Output Data Files}

Hierarchical Data Format (HDF) is a reasonably widespread format used to create portable data files. The HDF system originated at and is currently supported by NCSA in Illinois. When 1 hdf $=. t$, XPLOTGIN will write the following 3D data sets in $\mathrm{HDF}$ format: instantaneous $P(z, t)$ and normalized ( $\equiv P(z, t) /<P(z)>$ ) radiation power, instantaneous $b(z, t)$ and normalized e-beam bunching data, and instantaneous $P(\lambda, z)$ and normalized radiation power spectra $(\equiv P(\lambda, z) /<P(z)>)$. Each of the aforementioned power diagnostics has units of watts/bin. HDF files may be generated in addition to either ASCII- or SDDS-format data files mentioned in the previous two sections. The postprocessor HDF capability is targeted toward version 3.4 of the library and is thus seriously out of date as of late 2001 .

\subsection{Generating Wiggler Exit, Radiation Field Dump Files}

For various reasons it can be useful to generate an output file containing the time-dependent radiation fields at the wiggler exit. There are two methods to do this at present. For a fully poly- 
chromatic run, 1_write_outfld=.t will generate an ASCII file for the complex $\hat{E}(r, t)$ named run name.fld (e.g. palac.fld). The file will also contain locations of the radial grid points and a normalization value for the electric fields (GINGER internally scales electric field values). One can use this file to propagate the time-dependent radiation field to the far field and/or an intermediate position. One can override the default $z$-location of the wiggler exit by also inputting a value in meters for the parameter $\mathbf{z}_{\text {_write_outfld. }}$

A second possibility is applicable to oscillator mode runs only and was developed for the specific case of an oscillator-radiator configuration. If $l_{\text {_write_flde.t }}$, the postprocessor dumps the wiggler exit radiation electric field information from oscillator pass number npass_w. If not input in the preferences file, npass_w defaults to npass. The format at present is that used by GINGER for restart field files and is sufficiently obscure and non-robust that users should not attempt to use it for anything other than a oscillator or radiator restart run.

\subsection{Generating $z$-dependent, Single-Slice, Radiation Field Dump Files}

Knowledge of the $z$-dependent radiation field advancing (due to slippage) beyond a given electronbeam slice permits one to propagate a subsequent (actually preceding in time) slice. As explained in $\S 3.4 .4$, one can use the field information from a polychromatic run to initialize a single-slice Fredmode run with detailed output macroparticle diagnostics. To create such a field_file from a pltfile, in the preferences file namelist one should set the input variable $\mathbf{l}_{\text {_write_fld_z }}=. t$. If output from other than the "head" electron beam slice is wanted, the variable nslice_fld_z should be set to an integer value in the interval 1 to nside with 1 corresponding to the tail slice and nside to the head slice. Note, if the pltfile had $\mathbf{n} \_$diag_mod $\neq 1$, this feature will not work properly in a restart run (due to missing $z$-location field information). The output field file will be in SDDS format and named run_name-fld__z.sdds (e.g. palac-fld_z.sdds).

\subsection{Macroparticle Phase Space Plot Control Variables}

At present, macroparticle scatterplots are only available only for single-slice FRED-mode runs whose GINGER input file had nspec $\geq 1$. The scatterplots will automatically be produced unless the variable 1_scatterplots=.f. The 2-element array gamma_limit specifies the lower and upper limits in $\gamma$ for the $\gamma-\theta$ scatterplots - this option is useful if one is attempting to generate a movie. 1_plotMeV = .t sets the ordinate scale in the $\gamma-\theta$ plots to be megavolts rather than the default of Lorentz factor. 1_plot_gammap=. $t$ substitutes the computed variable $\gamma_{\|} \equiv\left(1-\beta_{z}^{2}\right)^{1 / 2}$ for $\gamma$ in the scatterplots. This option can be useful when examining the performance of a highly tapered wiggler. Setting 1_plot_xy=.t will generate $x-y, x-p_{x}, \quad$ etc. scatterplots, permitting the user to check the $z$-dependent evolution of the e-beam transverse profile in the focusing lattice. 


\subsection{Default Values for Preference File Namelist Variables}

In general, logical variables in the preference file namelist are normally of the form 1 name where name represents some mnemonic for a plot function or whatever, integer variables start with the letters $i-n$, and real variables start with the remaining letters of the alphabet. The default values for \&inpref namelist variables are as follows:

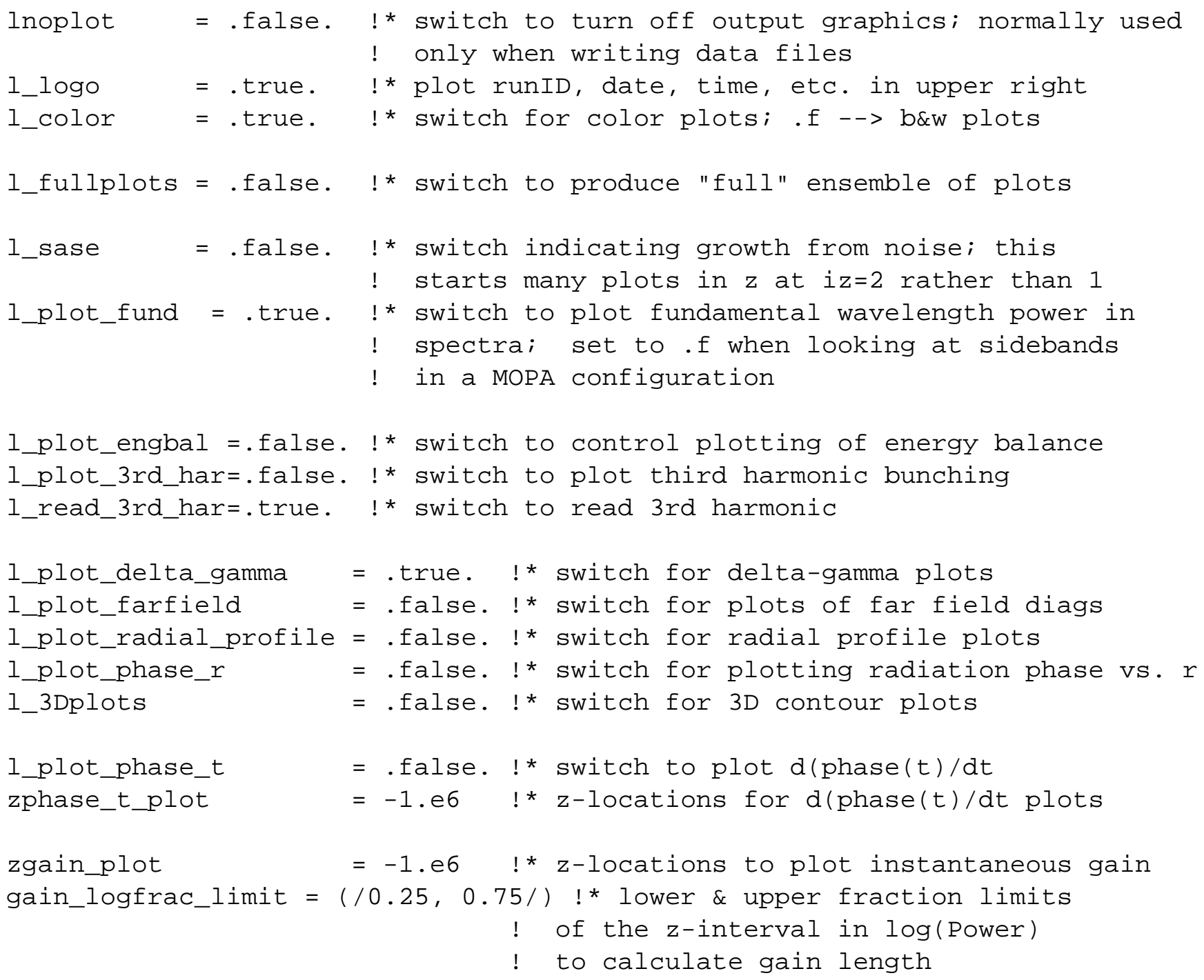









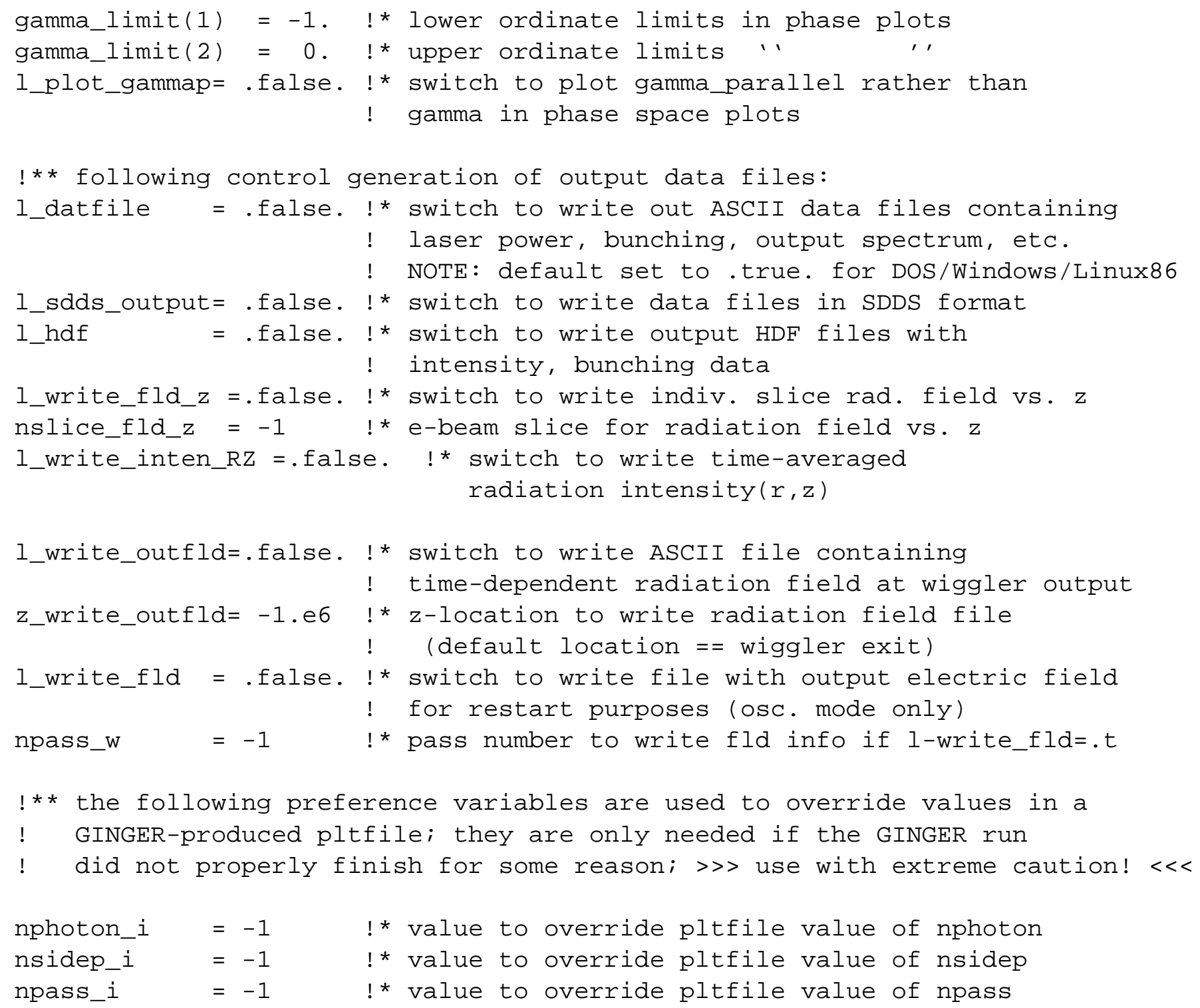




\section{The Physics Model of GINGER}

GINGER uses various approximations, assumptions and physics models when simulating the behavior of free-electron lasers. This section discusses these issues and some others related to numerical accuracy.

\subsection{Application of the Paraxial Wave Equation}

GINGER adopts the slowly-varying envelope approximation (SVEA), which is also widely known as either the paraxial wave equation or the eikonal approximation. GINGER applies this approximation by separating the local radiation electric field $E(r, z, t)$ into a product of a "fast" modulation $\exp \left[i\left(k_{s} z-\omega_{o} t\right)\right]$ times a "slow" envelope modulation $\tilde{E}(r, z, t)$, whose $r$ and $z$ spatial derivative scale lengths are much greater than $\lambda_{s}$ and whose time derivative is much slower than $\omega_{o}$.

The slow modulation $\tilde{E}$ is a complex quantity whose spatial and temporal phase $\phi(r, z, t)$ is measured relative to that of hypothetical plane wave whose phase varies exactly as $\left(k_{s} z-\omega_{o} t\right)$. In GINGER, an individual macroparticle's longitudinal phase $\theta$ is also measured relative to this hypothetical plane wave. The "FEL" ponderomotive phase $\psi$ of a macroparticle is then $\psi \equiv \theta+\phi$. Effects of diffraction, gain, and refraction all lead to $\phi$ becoming non-zero, while a non-resonant macroparticle energy leads to a monotonic increase or decrease of $\theta$ with $z$.

Neglecting the second $z-$ and $t$ - derivatives of $\tilde{E}$ relative to $-k_{s}^{2} \tilde{E}$ and $-\omega_{o}^{2} \tilde{E}$ respectively, the full wave equation may be approximated as

$$
2 i\left(k_{s} \frac{\partial \tilde{E}}{\partial z}+\omega_{0} \frac{\partial \tilde{E}}{\partial t}\right)+\nabla_{\perp}^{2} \tilde{E}=-\frac{4 \pi i}{c^{2}} \omega_{0} \tilde{J}_{\perp}+\left(\frac{\omega_{o}^{2}}{c^{2}}-k_{s}^{2}\right) \tilde{E}
$$

Here $\tilde{J}_{\perp}$ is the bunched component of the transverse e-beam current and, for non-waveguide cases, $\omega_{0}=c k_{s}$ and $\partial \omega / \partial k \equiv c$. The above equation may be rearranged to produce

$$
\frac{1}{c} \frac{\partial \tilde{E}}{\partial T}=\frac{i}{2 k_{s}} \nabla_{\perp}^{2} \tilde{E}-\frac{2 \pi}{c} \tilde{J}_{\perp}
$$

The operator $c^{-1} \partial / \partial T$ represents the co-moving derivative in the forward direction in the wiggler.

For nearly all "normal" FEL problems, the scaling $\nabla_{\perp}^{2}, \partial^{2} / \partial z^{2} \ll k_{s}^{2}$ is well satisfied since Rayleigh ranges and gain lengths are much longer than $\lambda_{s}$. The temporal part of the SVEA, which asserts $\left|\partial^{2} \tilde{E} / \partial t^{2}\right| \ll \omega_{o}^{2} \tilde{E}$, is similarly justified for FEL amplifiers whose gain bandpass is small compared to $\omega_{o}$. The approximation begins to fails if one is interested in a device whose spectral width is comparable to $\omega_{o}$, as might be true if one is examining incoherent spontaneous emission over an extremely broad bandpass. 


\subsection{Application of the KMR Equations}

In order to determine the value of the bunched transverse electron current and to advance the macroparticles's energies and longitudinal phases, GINGER adopts the Kroll-Morton-Rosenbluth (KMR) (IEEE J. Quantum Elec., QE-17, pp. 1436-1468, 1981) wiggle-period-averaging approximation. In this approximation, details of the so-called quiver (or "figure 8") motion and the $v_{\perp} \cdot \tilde{E}_{\perp}$ wave-macroparticle interaction are averaged over one or more wiggler wavelengths $\lambda_{w}$ in $z$. The latter averaging requires that $\gamma, a_{w}, \lambda_{w}$, and $\tilde{E}_{\perp}$ be constant or slowly varying over a wiggler wavelength. Furthermore, FEL radiation is presumed to be emitted exactly in the forward direction. Nearly all FEL's have gain lengths and Rayleigh ranges much longer than $\lambda_{w}$ and have wiggler errors small compared to a locally averaged $a_{w}$. Hence, the KMR approximation is expected to be excellent. Numerically, GINGER evaluates values for macroparticles, the radiation field, and the wiggler field locally in space as opposed, for example, of trying to use some average value of an interval $\Delta z=\lambda_{w}$. Note that the KMR equations generally assert a much slower variation in $z$ than does the SVEA for the radiation field.

\subsection{GINGER's Transverse Macroparticle Mover}

GINGER's transverse particle mover is that of its monochromatic predecessor FRED and is fully three-dimensional and relativistic. The mover uses a 4th-order Runge-Kutta algorithm and follows the wiggler-period-averaged betatron motion only, and not the "fast" wiggle motion (as is appropriate for GINGER's adoption of the KMR equations). At present, the particles are advanced at the same $z$-locations as the radiation field, which typically involves a step size generally smaller than $\lambda_{w}$ which itself is much smaller than the betatron wavelength in either plane. Hence, the numerical error in betatron motion is expected to be extremely small. As of late 2001, transverse drift terms due to wiggler errors can be included via lattice file's generated by the XWIGERR program (see §3.5.9). Longitudinal space charge forces are also evaluated over each field advance and are applied within the KMR $\gamma$ advance of the individual macroparticles.

\subsection{Temporal Structure of GINGER}

Following the approach pioneered by the work of W. Colson and D. Quimby, GINGER models the "slow" temporal modulation $\tilde{E}$ of the "complete" transverse electromagnetic field and particle transverse current source terms by resolving the radiation field and the particle beam into discrete, equally spaced transverse slices. In GINGER, the temporal separations between adjoining slices is normally many times $\lambda_{s} / c$, especially for FEL's whose Pierce parameter $\rho \ll 10^{-2}$. Thus, the macroparticles and radiation field which belong to a given transverse slice should be thought of those of a typical, "sample" ponderomotive well in the temporal center of a the slice. Following this 
ansatz, dynamically, when a given macroparticle's longitudinal phase crosses one of the ponderomotive well boundaries $\theta= \pm \pi$, the particle instantly reappears at the other boundary (as opposed to jumping into a well of an adjacent slice). This treatment should work well for spectral bandpasses small compared to the central wavelength $\lambda_{s}$ (i.e. each slice is many $\lambda_{s} / c$ wide temporally) but will break down as full bandpass $\Delta \lambda$ becomes comparable to the central wavelength $\lambda_{s}$.

Although some workers conceive of the electron beam and radiation slices in a polychromatic FEL code as being separated in space (as in a hypothetical snapshot in time), the correct picture (for GINGER at least) is a separation in time (as in an oscilloscope trace). Otherwise, one could not properly model very high gain FEL's whose gain length is less than the equivalent length of the electron beam modeled in the code. Within GINGER itself, only the temporal modulation of the field is followed; decomposition into frequency components is done only by the postprocessor which uses FFT's to determine the frequency content of the electromagnetic field at any position in $(r, z)$.

\subsection{Discrete Slippage Model}

In optical FEL's, light moves forward with a phase and group velocity $c$ whereas the electron beam particles move forward with a longitudinal velocity $v_{\|} \approx k_{s} /\left(k_{s}+k_{w}\right)<c$. This velocity difference introduces a "slippage" between the light and resonant electrons of one radiation wavelength $\lambda_{s}$ for every wiggler period of propagation. In GINGER, this slippage is approximated in the following way: A given electron beam slice interacts with a single radiation slice for a discrete distance $\Delta z_{\text {interact }} \equiv L_{w} /$ nsidep. At the end of this interaction distance, the given electron beam slice abruptly "falls back" in time to begin interacting with a new radiation slice (here time is measured back from the head of the radiation pulse).

Due to considerations of computer memory management on non-MPP platforms (a problem far more pressing in 1985 when GINGER was first written than it is today), the code actually follows a given slice of beam particles through the entire wiggler, slipping it back in time relative to the radiation slices at the appropriate discrete positions in $z$. With this choice, it was possible to structure the code such that one and only only one set of macroparticles need be in memory at any given instant. Apart from some initial disk storage of particle phase space coordinates both at the beginning and end of the simulation run necessary when periodic boundary conditions are used, no disk swapping of particle quantities is required during the main body of the run. When using multiple processors in SMP mode, there is some disk/memory swapping throughout the run. On MPP's when using multiple processors, all the particles are simultaneously in memory and all the slices are advanced simultaneously, lockstep in $z$.

As mentioned above, at any given position $z$ the beam slice interacts with one and only one radiation slice. This ensures that information can move only from the front of the radiation beam to the back and not visa versa (excepting the effects of the periodic boundary conditions). This is 
a noteworthy difference from some other polychromatic FEL codes where the field quantities are interpolated in time to the particle positions and, similarly, the particle source terms are interpolated to the field positions. This alternative choice, in principle at least, can allow some information to move unphysically (i.e. faster than $c$ ) from the tail of the radiation field toward the head.

\subsection{Temporal/Frequency Window Duration and Resolution Considerations}

There are two ways that one may then set the duration $W$ of the simulation window in time: The first is to specify the equivalent length $c W$ using the input parameter window in meters. The second and more common way is to specify the parameter nsidep which is defined as the number of photon slices that will slip over a given e-beam slice over the duration of the wiggler. One should also note that as nsidep approaches $N_{w}$, the polychromatic bandpass begins to include "slow" time variations whose frequencies are becoming an appreciable fraction of $\omega_{o}$. This violates the neglect of the second time derivatives of $\tilde{E}$ which underlies the slowly-varying envelope approximation (SVEA) used in the field equations. Moreover, the KMR wiggle-period-averaged equations also require nsidep $\leq N_{w}$ to be true.

There are a number of physics issues to consider when attempting to choose "good" values of nsidep and nphoton. First, for a single pass amplifier, one wants the interaction distance

$$
\Delta z_{\text {interact }} \equiv L_{w} / \text { nsidep } \equiv \lambda_{w} \times\left[\text { window } /\left(\text { nphoton } \times \lambda_{s}\right)\right]
$$

between a given optical and e-beam slice to be a relatively small fraction (e.g. $\leq 25 \%)$ of the electric field gain length, which is approximately $\lambda_{w} / 4 \pi \rho$. Equivalently then,

$$
\text { nsidep } \geq 16 \pi \rho N_{w}
$$

At equality, the full normalized frequency bandpass,

$$
\left(\omega_{\max }-\omega_{\min }\right) / \omega_{o} \equiv \mathrm{nsidep} / N_{w}=16 \pi \rho
$$

should be more than sufficient to enclose the normalized gain bandpass whose full width $\sim 2 \rho$. Since $\rho$ typically lies in the range $10^{-2}$ to $10^{-4}$, nsidep is almost always significantly less than $N_{w}$. For cases beyond saturation where sidebands become important and/or where for one reason or another the peak of the gain curve has a frequency chirp with $z$ or time, one must ensure nsidep is sufficiently large.

On the other hand, one probably wants reasonably good frequency resolution $\Delta \omega$ within the gain curve. Since

$$
\Delta \omega / \omega_{o}=\lambda_{s} / \text { window }=\operatorname{nsidep} /\left(N_{w} \times \text { nphoton }\right)
$$


choosing $\Delta \omega / \omega_{o} \leq \rho / 2$ is equivalent to

$$
\text { nphoton } \geq 32 \pi\left[\text { nsidep } /\left(16 \pi \rho N_{w}\right)\right]
$$

Consequently, requiring a good $z$ resolution of the field gain length together with reasonable frequency resolution of the gain bandpass will probably require nside $=$ nphoton $\geq 96$.

For a "standard" LCLS-like run where there are $\sim 15$ power gain lengths in the wiggler, one will generally pick nsidep $\geq 64$ and nphoton $=$ nside $\geq 128$. The CPU run time of the simulation will increase linearly with nside but have a much slower than linear increase with increasing nsidep.

Another consideration which tends to make one increase both nsidep and nside is the accuracy of GINGER's discrete slippage algorithm for frequencies which lie a significant portion of the frequency bandpass away from the central frequency $\omega_{0}$. For nsidep sufficiently large that $\Delta z_{\text {interact }} \leq L_{\text {gain }} / 8$ (where $L_{\text {gain }}$ is the power gain length), the frequency $\omega_{\text {peak }}$ corresponding to the peak of the exponential gain curve can be offset from the central frequency $\omega_{o}$ of the simulation as much as $\pm 15 \%$ of full simulation spectral bandpass without appreciable (e.g. $\geq 10 \%$ increase in gain length) unphysical effects. The useful spectral bandpass decreases linearly with nsidep. When modeling SASE devices one must be somewhat careful in one's choice of $\lambda_{s}$ for a given $a_{w}$ or visa versa if there is not good resolution in $z$ of an exponential gain length. Otherwise, the predicted gain will be unphysically suppressed. 